\title{
Biorelevant two-stage in vitro testing for rDCS classification and in PBPK modelling - Case example ritonavir
}

Tom Fiolka ${ }^{1}$, Jens Van Den Abeele ${ }^{2}$, Patrick Augustijns ${ }^{2}$, Sumit Arora ${ }^{3}$, Jennifer Dressman $^{1,4}$

${ }^{1}$ Department of Pharmaceutical Technology, Goethe University, Frankfurt/Main, Germany

${ }^{2}$ Drug Delivery \& Disposition, KU Leuven, Leuven, Belgium

${ }^{3}$ Certara UK Limited, Simcyp Division, Sheffield, UK

${ }^{4}$ Fraunhofer Institute for Molecular Biology and Applied Ecology IME, Project Group

Translational Medicine and Pharmacology TMP, Frankfurt/Main, Germany

Address correspondence to Prof. Dr. Jennifer Dressman

Department of Pharmaceutical Technology, Goethe University

Max von Laue Str. 9, 60438 Frankfurt/Main, Germany

Tel.: +496979829680

Email: dressman@em.uni-frankfurt.de 


\begin{abstract}
Biorelevant two-stage in vitro testing is increasingly used as a tool for various applications in drug development. Three important applications of two-stage in vitro testing are the classification of weakly basic drug compounds as part of the "refined Developability Classification System" (rDCS), the prediction of intraluminal drug concentrations in the gastrointestinal tract and the prediction of plasma concentration profiles using Physiologically Based Pharmacokinetic (PBPK) modeling. For the weakly basic, antiretroviral drug ritonavir, two-stage testing is triggered as a customized investigation in the rDCS classification process to assess whether the drug could supersaturate and precipitate when exposed to the steep change in $\mathrm{pH}$ that occurs during drug transfer from the stomach into the small intestine. It was shown that for two Norvir ${ }^{\circledast}$ formulations, a tablet and an oral powder formulation, the two-stage test yielded similar results to the more complex "transfer" model with regard to the supersaturation and precipitation behavior of these amorphous solid dispersion formulations. Furthermore, solubility and two-stage data were mechanistically modelled in the in vitro data Analysis Toolkit (SIVA) and the results used as input parameters for a PBPK model built in the Simcyp Simulator.
\end{abstract}

Abbreviations:

rDCS, refined Developability Classification System; PBPK, Physiologically based pharmacokinetic modelling; FaSSGF, Fasted state simulated gastric fluid; FaSSIF, Fasted state simulated intestinal fluid; SGF, Simulated Gastric Fluid; SIF, Simulated Intestinal Fluid;

Keywords: Dissolution; in vitro models; Developability; Biopharmaceutical classification system (BCS); Physiologically Based Pharmacokinetic (PBPK) modeling; SimCyp PBPK modeling; in silico modeling; Amorphous Solid Dispersions (ASD); Precipitation; Supersaturation 


\section{Introduction}

The proportion of commercially available drugs on today's market as either weak bases or their salts is currently estimated to be $75 \% .{ }^{1}$ Poorly soluble, weakly basic drugs are, by their nature, prone to precipitation after entering the small intestine and their absorption from the intestine can be hindered by this process. The formulation of weakly basic drug candidates to promote supersaturation while preventing drug precipitation - in so-called enabling formulations - has become an important goal in drug development. With the increasing costs of drug development, which are now estimated to be over two and a half billion US dollars for just one drug, and an average time span of 12 years to reach the market, simple, fast and effective in vitro tests which can prospectively and correctly assess the in vivo performance of weakly basic drug candidates and their formulations are in great demand ${ }^{2,3}$. Experimental two-stage setups (exposing the drug to a change in environment representing the passage out of the stomach into the small intestine) such as the "transfer" model to evaluate the risk of possible in vivo drug precipitation have therefore become indispensable tools in the development of oral drug products ${ }^{4}$.

In the recently introduced refined Developability Classification System (rDCS), which is based on the former DCS by Butler and Dressman, customized two-stage in vitro tests for poorly soluble weakly basic drugs are used to classify compounds regarding their potential in vivo drug performance ${ }^{5-7}$. The authors stated that the in vivo performance of weakly basic drugs would be overpredicted if only dissolution under gastric conditions is used as an indicator. On the other hand, the in vivo performance would be underpredicted if only dissolution under intestinal conditions is taken into consideration, leading to false rejection of potential drug candidates in drug development. To avoid these misinterpretations, the rDCS proposes simple and robust two-stage experimental setups to gain valuable insight into the dissolution/precipitation behavior of weakly basic drug compounds in the upper gastrointestinal tract.

Following completion of a ring study by the OrBiTo (www.orbito-project.eu ) consortium, in which 16 research groups from both industry and academia participated in a study to validate a simplified twostage setup for in vitro testing, a second ring study was recently published by Berben et al. ${ }^{8,9}$ The results of this second study, which was also conducted by several industrial and academic partners from the OrBiTo consortium, showed encouraging reproducibility and robustness of two-stage in vitro testing in various laboratories for the weakly basic drug indinavir. Furthermore, several authors have started to incorporate the results of various two-stage tests in vitro into PBPK in silico programs to build models that are able to predict the in vivo luminal and systemic behavior of weakly basic drugs. ${ }^{10-13}$

The aim of this paper is to describe two different applications of two-stage in vitro testing for the antiretroviral drug ritonavir, on the one hand its application as a "customized investigation" for the rDCS, and on the other hand as a promising tool to optimize PBPK models for weakly basic, poorly soluble drugs and drug candidates.

\section{Experimental}

\subsection{Chemicals and reagents}

Ritonavir active pharmaceutical ingredient was purchased from VWR (Leuven, Belgium). Norvir ${ }^{\circledR}$ $100 \mathrm{mg}$ tablets and Norvir ${ }^{\circledast} 100 \mathrm{mg}$ oral powder were purchased from Phoenix (Hanau, Germany). 
Sodium hydroxide and sodium dihydrogen phosphate monohydrate were purchased from Merck KGaA (Darmstadt, Germany). Sodium chloride, hydrochloric acid (37\%) and acetic acid were purchased from VWR (Leuven, Belgium). Methanol (HPLC grade) was purchased from Merck KGaA. FaSSIF/FeSSIF/FaSSGF powder, FaSSIF buffer concentrate and FaSSGF buffer concentrate was kindly donated by biorelevant.com (London, UK).

\subsection{Biorelevant and compendial media preparation}

Biorelevant media were prepared following instructions from biorelevant.com. FaSSGF was obtained by initially preparing FaSSGF buffer using "FaSSGF buffer concentrate" and subsequently adding FaSSIF/FaSSGF/FeSSIF powder. FaSSIF was prepared in a similar manner but using the "FaSSIF buffer concentrate". FaSSIFx2 pH 7.5 (double concentrated) was prepared as previously described by Mann et al. ${ }^{8}$. Further FaSSIF media with varying bile salt concentrations were prepared using the "FaSSIF buffer concentrates" and then adding different amounts of FaSSIF/FaSSGF/FeSSIF powder. Simulated Gastric Fluid (SGF) and Simulated Intestinal Fluid (SIF) were prepared following instructions by the United States Pharmacopeia $41^{14}$. Double concentrated simulated intestinal fluid (SIF) was obtained by dissolving double the amounts of potassium dihydrogen phosphate and sodium hydroxide required for SIF and subsequently adjusting the solution to $\mathrm{pH} 7.5$. After combining gastric and intestinal stages of two stage experiments, the composition corresponded to that of Simulated Intestinal Fluid (pH 6.8) or FaSSIF (pH 6.5), respectively.

Table 1

Composition of biorelevant media used in ritonavir experiments

\begin{tabular}{|c|c|c|c|c|c|c|}
\hline & $\begin{array}{l}\text { FaSSIF- } \\
\text { Buffer }\end{array}$ & $\begin{array}{l}\text { FaSSIF-V1 } \\
(0.5 \mathrm{x})\end{array}$ & $\begin{array}{l}\text { FaSSIF-V1 } \\
(1 \mathrm{x})\end{array}$ & $\begin{array}{l}\text { FaSSIF-V1 } \\
(1.5 \mathrm{x})\end{array}$ & $\begin{array}{l}\text { FaSSIF-V1 } \\
\qquad(2 x)\end{array}$ & FaSSGF \\
\hline \multicolumn{7}{|l|}{ Buffer } \\
\hline Sodium chloride [mM] & 105.85 & 105.85 & 105.85 & 105.85 & 105.85 & 34.2 \\
\hline Hydrochloric acid [mM] & - & - & - & - & - & 25.1 \\
\hline $\begin{array}{l}\text { Sodium dihydrogen } \\
\text { phosphate }[\mathrm{mM}]\end{array}$ & 28.36 & 28.36 & 28.36 & 28.36 & 28.36 & - \\
\hline Maleic acid [mM] & - & - & - & - & - & - \\
\hline Sodium hydroxide [mM] & 8.7 & 8.7 & 8.7 & 8.7 & 8.7 & - \\
\hline \multicolumn{7}{|l|}{ Biorelevant excipients } \\
\hline Sodium taurocholate [mM] & - & 1.5 & 3.0 & 4.5 & 6.0 & 0.08 \\
\hline Lecithin [mM] & - & 0.375 & 0.75 & 1.25 & 1.5 & 0.02 \\
\hline
\end{tabular}

\subsection{Thermodynamic solubility experiments}

Solubility experiments for ritonavir (crystalline form) were conducted to estimate the gastric and intestinal solubilities of the drug using compendial media (SGF with a pH value of 1.2 and SIF with a $\mathrm{pH}$ value of 6.8) and biorelevant media (FaSSGF pH 1.6 and FaSSIF pH 6.5). Further, the solubility was measured in an acetate buffer representing elevated $\mathrm{pH}$ gastric conditions $(\mathrm{pH} 4.0)$. Uniprep ${ }^{\circledR}$ 
systems (Whatman Inc., Sanford, ME, USA) were utilized for solubility determinations by introducing an excess amount of drug compound into each vial and consequently adding $3 \mathrm{~mL}$ of media. Vials were closed with Uniprep ${ }^{\circledR}$ caps and incubated on an orbital shaker at $37^{\circ} \mathrm{C}$ for 24 hours. After 24 hours, samples were filtered through the built-in Uniprep ${ }^{\circledR} 0.45 \mu \mathrm{m}$ PTFE filter and the filtrates were analyzed by HPLC. Solubility experiments in each medium were performed in triplicate.

\subsection{Single-stage dissolution experiments}

For single-stage and two-stage experiments, a calibrated USP 2 apparatus (Erweka DT 720, Heusenstamm, Germany) was employed. Traditional USP 2 sampling cannulas were replaced by specially designed cannulas manufactured by AstraZeneca to facilitate sampling from the gastric media, due to the low media volume of $250 \mathrm{~mL}$. In addition, cannula filters were attached to each cannula to ensure that no solid drug was removed from vessels during sampling. The paddle speed was set at $75 \mathrm{rpm}$, and the temperature of the medium was maintained at $37 \pm 0.5^{\circ} \mathrm{C}$. For singlestage testing, a volume of $250 \mathrm{~mL}$, representing the gastric environment $(50 \mathrm{~mL}$ residual volume together with $200 \mathrm{~mL}$ of water administered with the drug formulation), or $500 \mathrm{~mL}$ of medium simulating the small intestine was used. Samples were taken manually after 5, 10, 20, 30, 45, 60 and 90 minutes into a $5 \mathrm{~mL}$ plastic syringes through a stainless steel cannula fitted with a $10 \mu \mathrm{m}$ polyethylene cannula filter, whereby the first $4 \mathrm{~mL}$ of sampling medium was returned through a 0.45 $\mu \mathrm{m}$ PTFE syringe filter (Whatman $\mathrm{GmbH}$, Dassel, Germany) to the dissolution vessel. The residual volume was transferred through the filter into an Eppendorf Cap and $100 \mu \mathrm{L}$ of filtrate was immediately diluted with mobile phase 1:10 to prevent precipitation of the drug in the sample. All experiments were conducted with $\mathrm{n}=6$.

\subsection{Two-stage dissolution experiments}

In the first stage of the experiment, the dosage form was allowed to disintegrate and dissolve for 30 minutes in the gastric medium (SGF or FaSSGF). At 30 minutes, $250 \mathrm{~mL}$ double concentrated SIF or FaSSIF ( $\times 2$ ) was added to the gastric medium by pouring it into the gastric medium from a volumetric flask over a period of maximum 1 second i.e. "dumping". To add the concentrate, the lid of the dissolution vessel was temporarily lifted and immediately closed again after media addition. Samples were taken manually after $5,10,20$ and 30 minutes from the gastric phase. After media addition samples were taken at $35,40,45,60,90$ and 120 minutes after initiation of the experiment. Samples were handled in the same manner as described for single-stage dissolution experiments. All experiments were conducted with $n=6$. 




Fig. 1 Graphical representation of the simplified two-stage setup

\subsection{Transfer experiments}

The transfer model setup (Figure 1), which was first introduced by Kostewicz et al. in 2004, consists of two separate compartments representing a gastric (mini vessels and mini paddles) and an intestinal compartment (standard USP 2 vessels and paddles) ${ }^{4}$. The paddles were set to a revolution speed of $75 \mathrm{rpm}$ and the temperature was kept at $37 \pm 0.5^{\circ} \mathrm{C}$. A programmable ISMATEC MC-Process IP5 peristaltic pump was used to transfer the content of the gastric (donor) compartment into the intestinal (acceptor) compartment, to simulate gastric emptying. Tubing (ISMATEC neoprene) was used for each pair of vessels to transfer both dissolved and undissolved particles (IDEX Health \& Science $\mathrm{GmbH}$ ). As recommended by Ruff et al., first order transfer with a 9 min half-life was used for all transfer experiments to represent average physiological gastric emptying in the fasted state ${ }^{15}$. For better comparison among single-stage testing, simplified two-stage tests and the transfer model, volumes of $250 \mathrm{~mL}$ of FaSSGF and $250 \mathrm{~mL}$ of FaSSIF x 2 concentrate were applied to reach a final volume of $500 \mathrm{~mL}$ FaSSIF at a pH of $6.5 \mathrm{in}$ all experiments. Samples were taken manually from the acceptor vessel after 2, 5, 10, 20, 30, 35, 40, 45, 60, 90, 120 and 180 minutes. All samples were handled in the same manner as described for single-stage and two-stage dissolution testing. All transfer model experiments were performed in triplicate. 


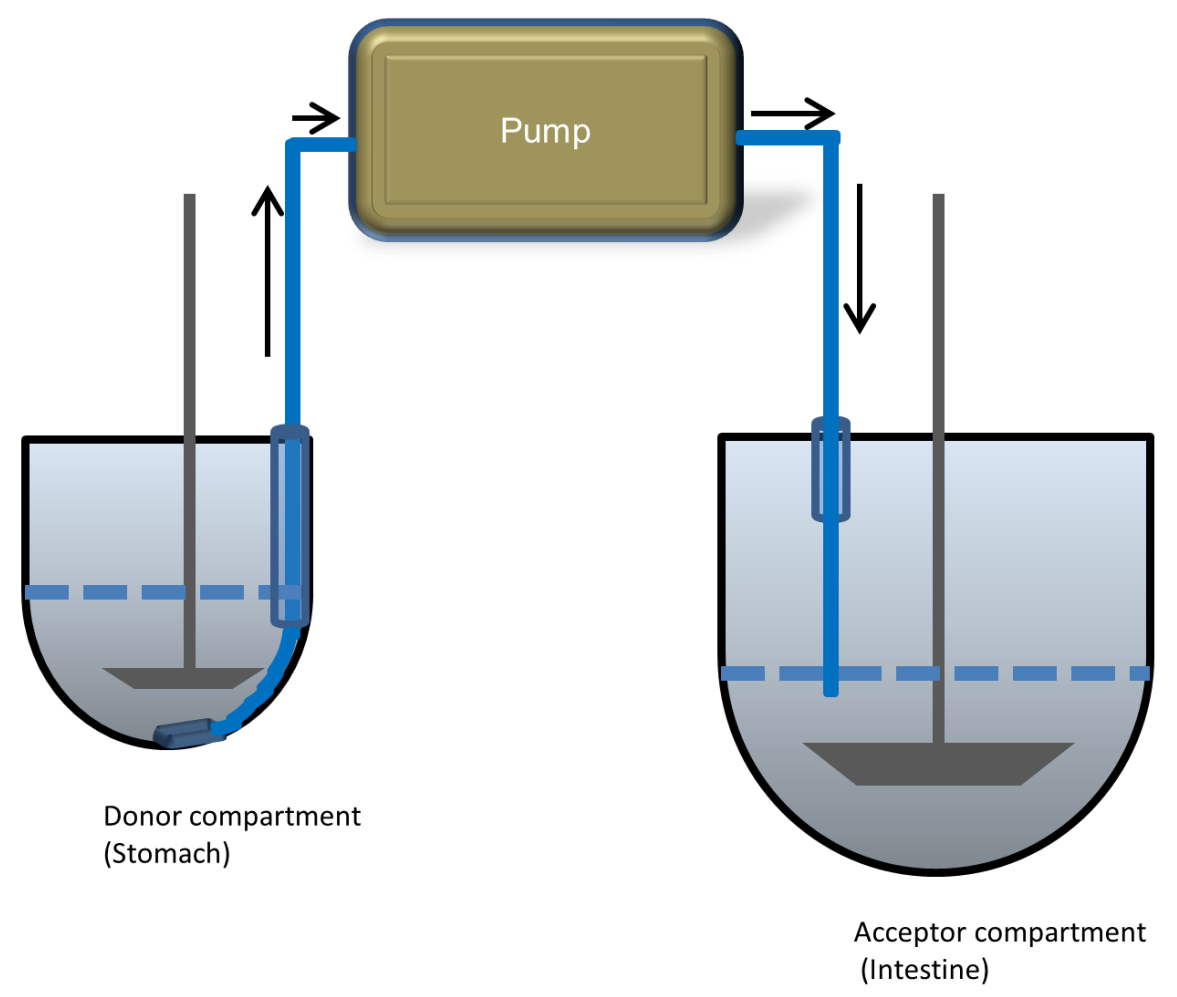

Fig. 2 Graphical representation of the transfer model

\subsection{Quantification by HPLC analysis}

Samples from all experiments were quantified by HPLC analysis using an end-capped Purospher ${ }^{\circledR}$ STAR RP-18 $(5 \mu \mathrm{m})$ column (Merck KGaA, Darmstadt, Germany). The HPLC system consisted of a Hitachi LaChrom Elite by VWR Hitachi using an L-2130 pump, an L-2300 column oven, an L-2200 auto sampler and an L-2400 UV detector. For evaluation of the resulting chromatograms EZChrom Elite software version 3.3.2 SP2 was used (VWR, Darmstadt, Germany). The mobile phase comprised methanol and a $25 \mathrm{mM}$ sodium acetate buffer in a ratio of $25: 75$, adjusted to a $\mathrm{pH}$ of 3.5 . The flow rate of the pump was $1.0 \mathrm{~mL} / \mathrm{min}$ and the injection volume was $50 \mu \mathrm{L}$. UV-VIS detection wavelength was set at $247 \mathrm{~nm}$ at $30^{\circ} \mathrm{C}$. The retention time of ritonavir was approximately 6.4 minutes and the limit of quantification was $0.2 \mu \mathrm{g} / \mathrm{ml}$. A calibration curve over the appropriate concentration range was prepared for each analytical run and all were linear with $a R^{2} \geq 0.9995$.

\subsection{Refined Developability Classification System (rDCS)}

The refined Developability Classification System (rDCS) builds on the framework of the DCS and $B C S$ to evaluate drugs and their formulations regarding suitability for oral administration. High absorption numbers $(A n)>1$ correspond to good permeability of the drug, leading to classification in class I or II, while high dose numbers (Do) $>1$ describe poor solubility, leading to classification in class II or IV. Class II is further divided into class IIa and IIb. Drugs in class Ila can be described as dissolution-limited drugs, making particle size of the compound an important factor in the absorption rate. The absorption of drugs in class Il ib solubility-limited, indicating that an enabling formulation is required to boost oral absorption (Figure 3). The rDCS distinguishes between standard investigations ( $\mathrm{SI}$ ) and customized investigations $(\mathrm{Cl})$, whereby customized investigations are only triggered in special cases. For class II drugs, Cls are used to differentiate between dissolution rate (Ila) and solubility limitations (IIb) to absorption. For weakly basic compounds, two-stage experiments are strongly recommended to detect possible supersaturation/precipitation issues. While supersaturation may lead to a shift of the compound into a more favorable characterization 
class due to the higher concentration of drug driving absorption, precipitation will work against this benefit.

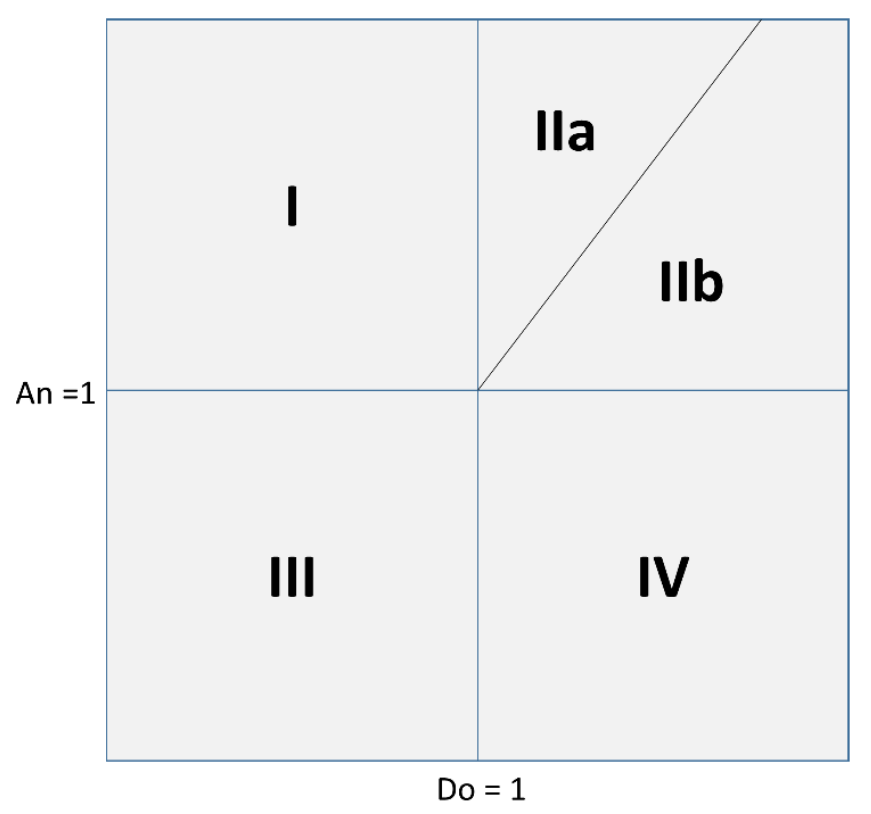

Fig. 3 Graphical representation of the refined Developability Classification System

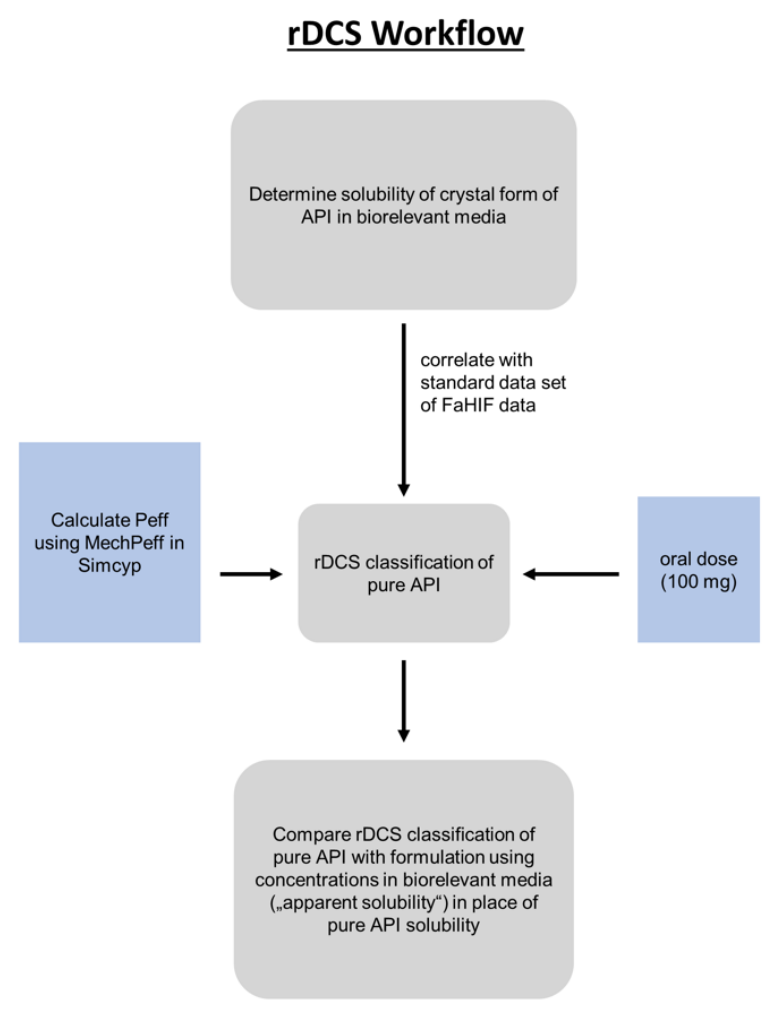

Fig. 4 Graphical representation of the rDCS workflow 


\subsection{Pharmacokinetic intraluminal and clinical data of ritonavir $100 \mathrm{mg}$ (Norvir ${ }^{\circledast}$ ) tablets}

Pharmacokinetic clinical study results were kindly provided by KU Leuven, Belgium ${ }^{16}$. Ritonavir $100 \mathrm{mg}\left(\mathrm{Norvir}^{\circledast}\right.$ ) tablets were administered to 5 healthy study participants ( 2 female and 3 male, age 25-31 years) together with $240 \mathrm{~mL}$ of water under fasted conditions. Luminal drug samples were taken as aspirates through a catheter from the duodenal lumen over the first five hours, and venous blood was sampled over eight hours.

Additionally, the mean profile of clinical data of 27 healthy volunteers (standard deviations for each sampling point given) from a separate study by $\mathrm{Ng}$ et al. was used to verify the in-house PBPK model 17.

\subsection{Pharmacokinetic clinical data of ritonavir $100 \mathrm{mg}$ (Norvir ${ }^{\circledR}$ ) oral powder}

Literature pharmacokinetic data from Salem et al. for a $100 \mathrm{mg}$ Norvir ${ }^{\circledR}$ oral powder (oral suspension) formulation was used to further verify the PBPK model ${ }^{18}$. This study investigated the pharmacokinetics of ritonavir after an oral administration of $100 \mathrm{mg}$ of an oral powder formulation for 24 fasted healthy volunteers. The results were presented as mean profiles with standard deviations. $C_{\max }$ and $A U C$ values were also reported.

\subsection{Statistical analysis}

For statistical comparison of predicted and observed in vivo plasma profile concentrations, $\mathrm{FE}$ (Fold error), AFE (Absolute Fold Error) and AAFE (Average Absolute Fold Error) were used according to Obach et al. ${ }^{19}$.

\subsection{SIVA (Simcyp In Vitro Data Analysis Toolkit)}

The SIVA ${ }^{\circledR}$ toolkit Version 3 was used to determine various Simcyp Simulator parameter inputs from in vitro experiments using the stepwise in vitro-in vivo extrapolation (IVIVE) approach described by Pathak et al. ${ }^{12,13}$. Using this approach, experimental solubility and dissolution results are analyzed mechanistically in SIVA to estimate / confirm intrinsic parameters that are essential for the simulation of in vivo drug absorption. Most of the input parameters required for the SIVA toolkit module "Solubility" were taken from the ritonavir model proposed by Arora et al. ${ }^{20}$. Exceptions were

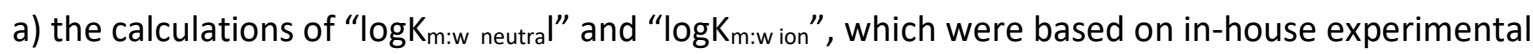
data in FaSSIF-V1 at various physiologically relevant bile salt concentrations, b) parameters defining supersaturation and precipitation behaviors, which were extracted from two-stage and transfer experiments. Instead of using a $K_{D}$ value (first order disintegration rate), a DLM (Diffusion Layer Model) scalar of 0.004 was calculated with the SIVA "Dissolution" function to characterize the dissolution behavior of the Norvir ${ }^{\circledR}$ tablet in the gastric test phase. For the Norvir ${ }^{\circledast}$ oral powder, a DLM scalar of 1 was applied due to the almost instantaneous dissolution of the amorphous powder.

\subsection{PBPK Simcyp ADAM (Advanced dissolution absorption and metabolism) model}

The PBPK Simcyp ${ }^{\circledR}$ ADAM model for Norvir ${ }^{\circledast} 100$ mg tablets was developed by Arora et al. ${ }^{20}$. The parameters used for setting up the PBPK Simcyp ADAM model in the Simcyp Simulator are shown in Table 2 . The initial model by Arora et al. was subsequently adapted to take the in-house experimental data into consideration. Experimental data were entered into the SIVA toolkit and the resulting parameters were then fed into the Simcyp Simulator. The license for the Simcyp Simulator V18 Release 2 and SIVA 3 Release 1 was kindly provided by Certara in form of a teaching license. 


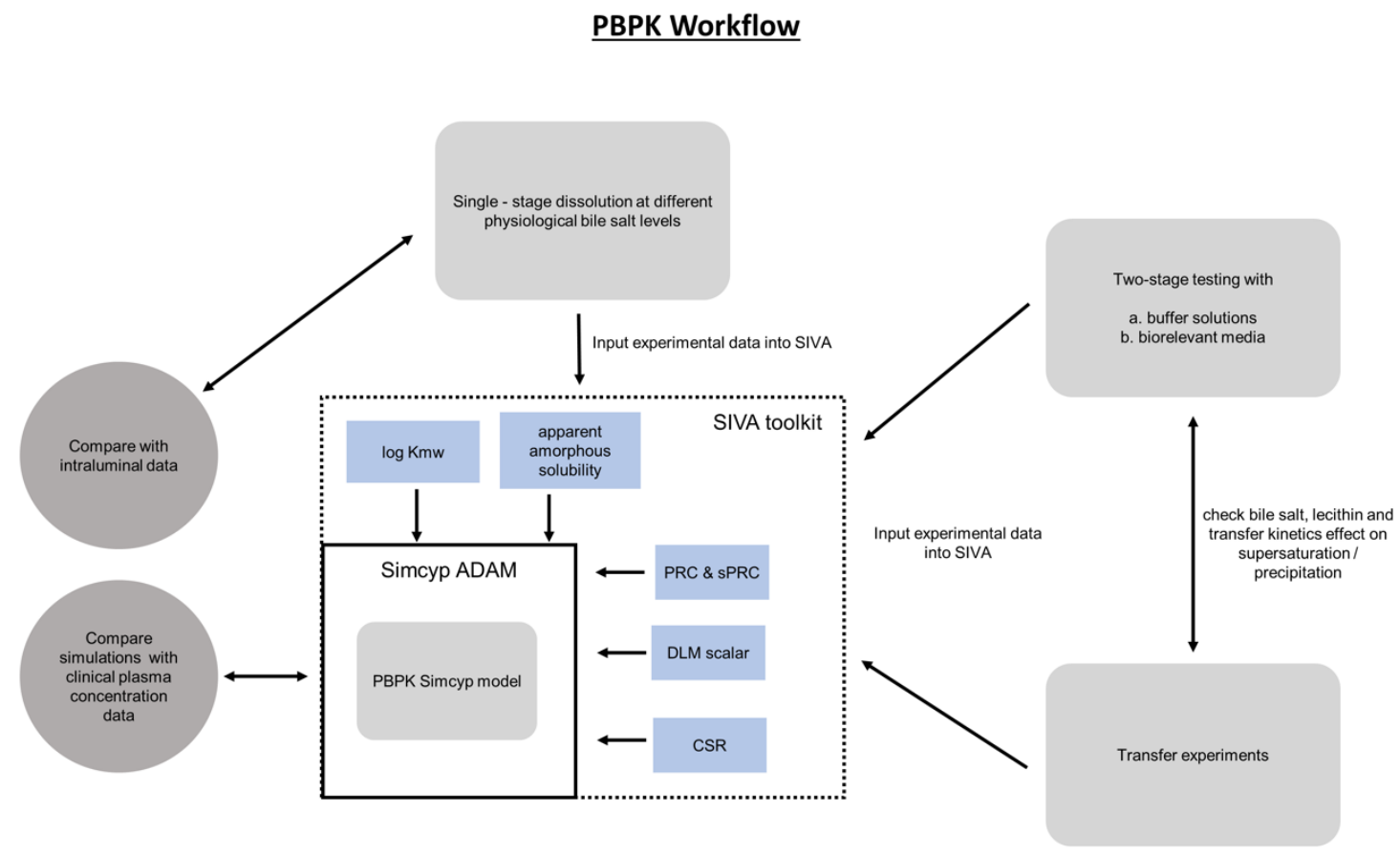

Fig. 5 Graphical representation of the PBPK workflow 
Table 2

Model input parameters used in the Simcyp Simulator (adapted from Arora et al.)

\begin{tabular}{|c|c|c|}
\hline Parameter & Value & Comment/Reference \\
\hline \multicolumn{3}{|l|}{ Physicochemical Properties } \\
\hline Molecular Weight & 721 & Law et al. 2001/2004 21,22 \\
\hline $\log P$ & 3.9 & https://pubchem.ncbi.nlm.nih.gov/compound/Ritonavir \\
\hline pKa (Diprotic Base) & $1.8,2.6$ & Law et al. 2001/2004 21,22 \\
\hline \multicolumn{3}{|l|}{ Absorption Related Parameters } \\
\hline Formulation & Immediate release & Amorphous solid dispersion formulation, Norvir ${ }^{\circ}$ tablet \\
\hline Particle Handling Method & PPB & Particle Population Balance \\
\hline Particle Size Distribution & Monodisperse & Assumed as data not available \\
\hline Particle Size $(\mu \mathrm{m})$ Monodisperse & 10 & Assumed as data not available \\
\hline Intrinsic Solubility (mg/mL) & 0.0385 & Xu et al. $2017^{23}$ \\
\hline Solubility Factor 1 (SF1, salt limiting solubility) & 103.90 & Fitted with SIVA 3, Law et al. 2004 21,22 \\
\hline \multicolumn{3}{|l|}{ Bile Micelle Partition Coefficient } \\
\hline $\log K_{m: w}$, neutral & $3.88(3.894 *)$ & Fitted with SIVA 3, Xu et al. $2017^{23}$ \\
\hline $\log K_{m: w}$, ion & NA & No ionization for ritonavir in proximal small intestine \\
\hline DLM scalar for stomach compartment & 0.004 & Fitted with SIVA 3 \\
\hline Precipitation Model & Model 2 & \\
\hline CSR & 1 & In-house (same as Xu et al. ${ }^{23}$; Ellenberger et al $2018^{24}$ ) \\
\hline $\operatorname{PRC}(1 / h)$ & 0.0001 & In-house (same as Indulkar et al. 2018 and 2019 25,26) \\
\hline $\operatorname{sPRC}(1 / \mathrm{h})$ & 1000 & In-house (same as Xu et al. $2017^{23}$ ) \\
\hline Particle heff prediction & Hintz-Johnson & \\
\hline MechPeff, $P_{\text {trans }, 0}\left(10^{-6} \mathrm{~cm} / \mathrm{s}\right)$ & 1000.57 & Predicted \\
\hline$P_{\text {eff,man }}(\mathrm{cm} / \mathrm{s})$ (Duodenum;Jejunum I-II) & $2.75 ; 7.25 ; 5.08$ & Predicted \\
\hline$P_{\text {eff,man }}(\mathrm{cm} / \mathrm{s})($ Ileum I-IV) & $1.13 ; 1.13 ; 1.12 ; 1.07$ & Predicted \\
\hline$P_{\text {eff,man }}(\mathrm{cm} / \mathrm{s})($ Colon) & 0.58 & Predicted \\
\hline Colon Abs Scalar & 0.1 & Fitted to recover terminal phase of ritonavir \\
\hline \multicolumn{3}{|l|}{ Distribution Related Parameters } \\
\hline$f_{u}$, plasma & 0.015 & Hsu et al. 199727 \\
\hline $\mathrm{V}_{\mathrm{sS}}(\mathrm{L} / \mathrm{kg})$ (Full PBPK) & 0.410 & Umehara et al. $2018{ }^{28}$ \\
\hline \multicolumn{3}{|l|}{ Elimination Related Parameters } \\
\hline \multicolumn{3}{|l|}{ CL(CYP2D6) - BD Sup } \\
\hline$V_{\max }$ & 0.93 & Koudriakova et al. 199829 \\
\hline $\mathrm{K}_{\mathrm{m}}$ & 1 & Koudriakova et al. 199829 \\
\hline ISEF & 0.75 & Simcyp simulation file for ritonavir \\
\hline \multicolumn{3}{|l|}{ CL(CYP3A4) - BD Sup } \\
\hline $\mathrm{V}_{\max }$ & 1.37 & Koudriakova et al. 199829 \\
\hline $\mathrm{K}_{\mathrm{m}}$ & 0.07 & Koudriakova et al. $1998{ }^{29}$ \\
\hline ISEF & 0.24 & Simcyp simulation file for ritonavir \\
\hline \multicolumn{3}{|l|}{ CL(CYP3A5) - BD Sup } \\
\hline$V_{\max }$ & 1 & Koudriakova et al. $1998{ }^{29}$ \\
\hline $\mathrm{K}_{\mathrm{m}}$ & 0.05 & Koudriakova et al. 199829 \\
\hline ISEF & 0.24 & Simcyp simulation file for ritonavir \\
\hline Additional HLM Clearance $(\mu \mathrm{l} / \mathrm{min} / \mathrm{mg})$ & 75 & Optimized \\
\hline Renal Clearance (L/h) & 0.53 & Denissen et al. 199730 \\
\hline \multicolumn{3}{|l|}{ Simulation Toolbox Settings } \\
\hline Use UBL Volumes & Yes & \\
\hline PPB Radius Rate Cap $(\mu \mathrm{m} / \mathrm{h})$ & 100 & \\
\hline
\end{tabular}

$\left({ }^{*}\right)$ values applied by Arora et al. 


\section{Results and Discussion}

\subsection{Equilibrium solubility experiments}

As expected, the crystalline form of ritonavir shows a pronounced $\mathrm{pH}$ dependent solubility. The solubility of the crystalline form of ritonavir in SGF $(\mathrm{pH}=1.2)$ and FaSSGF $(\mathrm{pH}=1.6)$, representing the $\mathrm{pH}$ of the fasted stomach, was relatively high $(383 \mu \mathrm{g} / \mathrm{ml}$ and $77.9 \mu \mathrm{g} / \mathrm{ml})$. In contrast, solubility in media with $\mathrm{pH}$ values above both ritonavir pK values (1.9 and 2.6) were very low. In the pH 4 acetate medium, representing the hypochlorhydric stomach (e.g. induced by concomitant intake of proton pump inhibitors), was $6.2 \mu \mathrm{g} / \mathrm{ml}$. In SIF ( $\mathrm{pH}=6.8)$ the solubility was even lower at $3.4 \mu \mathrm{g} / \mathrm{ml}$. In FaSSIF $\mathrm{V} 1(\mathrm{pH}=6.5)$ the solubility was somewhat higher $(11.5 \mu \mathrm{g} / \mathrm{mL})$ due to the influence of bile salts and lecithin, which are known to increase solubility of compounds with moderate to high lipophilicity (ritonavir $\log \mathrm{P}=3.9)^{31,32}$.

Table 3

Mean \pm SD equilibrium solubility of crystalline ritonavir in different media $(n=3)$

SGF $(\mathrm{pH}=1.2) \quad$ FaSSGF $(\mathrm{pH}=1.6) \quad$ Hypochlorhydric $(\mathrm{pH}=4) \quad \operatorname{SIF}(\mathrm{pH}=6.8) \quad$ FaSSIF $(\mathrm{pH}=6.5)$

crystalline

solubility

$328.2 \pm 6.2 \quad 77.9 \pm 0.8$

$6.2 \pm 0.4$

$3.4 \pm 0.2$

$11.5 \pm 2.1$

$(\mu \mathrm{g} / \mathrm{ml})$

\subsection{Single-stage dissolution experiments}

Dissolution experiments are able to give valuable insight into in vivo disintegration and dissolution behavior of different oral drug formulations. As previously proposed by Markopoulos and Andreas et al. different levels of biorelevance regarding the complexity of suggested test media should be considered according to drug characteristics, triggering the use of Level II biorelevant media for a weakly basic drug ritonavir with good permeability characteristics but poor solubility ${ }^{33}$.

With the use of recently introduced buffer concentrates to facilitate buffer preparation, paired with the use of "biorelevant triple F powder", which allows the preparation of FaSSGF, FaSSIF-V1 and FeSSIF from one and the same powder mixture, preparation of biorelevant media has become considerably less time consuming and increasingly convenient for pharmaceutical scientists, which further encourages the use of biorelevant media in in vitro dissolution testing.

Using the less physiologically relevant compendial buffer media for dissolution testing can lead to misinterpretation of drug/formulation characteristics. For example, drug release from Norvir ${ }^{\circledR}$ tablets and powder in SGF ( $\mathrm{pH}$ 1.2, representing the lower limit of gastric $\mathrm{pH}$ ) is complete, while in FaSSGF $(\mathrm{pH} 1.6)$, where the $\mathrm{pH}$ is more representative of the average gastric $\mathrm{pH}$ value (physiological range between 1-3), ${ }^{34,35}$ the release is not complete (see Figure 4).

Final ritonavir concentrations of single stage dissolution tests in FaSSIF-V1 of the $100 \mathrm{mg}$ Norvir $^{\circledR}$ tablet $(50 \mu \mathrm{g} / \mathrm{ml})$ and the Norvir ${ }^{\oplus}$ oral powder formulation $(56 \mu \mathrm{g} / \mathrm{ml})$ were found to be very similar to the solubility values of the amorphous form reported in the literature $(60 \mu \mathrm{g} / \mathrm{ml})^{25}$. Final concentrations in two-stage tests are subsequently referred to as "apparent amorphous solubility", in agreement with the plateau over an extended period of time observed by Xu et al. ${ }^{23}$. 
The dissolution profiles of the formulations in FaSSGF were also used to help build the PBPK model. Instead of entering the dissolution profiles directly in the PBPK model, the SIVA toolkit was used to mechanistically model the dissolution profile, resulting in a DLM (Diffusion layer model) scalar value of 0.004 for the Norvir ${ }^{\circledR}$ tablet formulation in FaSSGF. This value was subsequently used as an input parameter to the PBPK model to represent in vivo gastric disintegration and dissolution in the stomach compartment. For the Norvir ${ }^{\circledR}$ oral powder formulation the DLM scalar was set at 1 due to the almost immediate drug release (within 5 minutes).
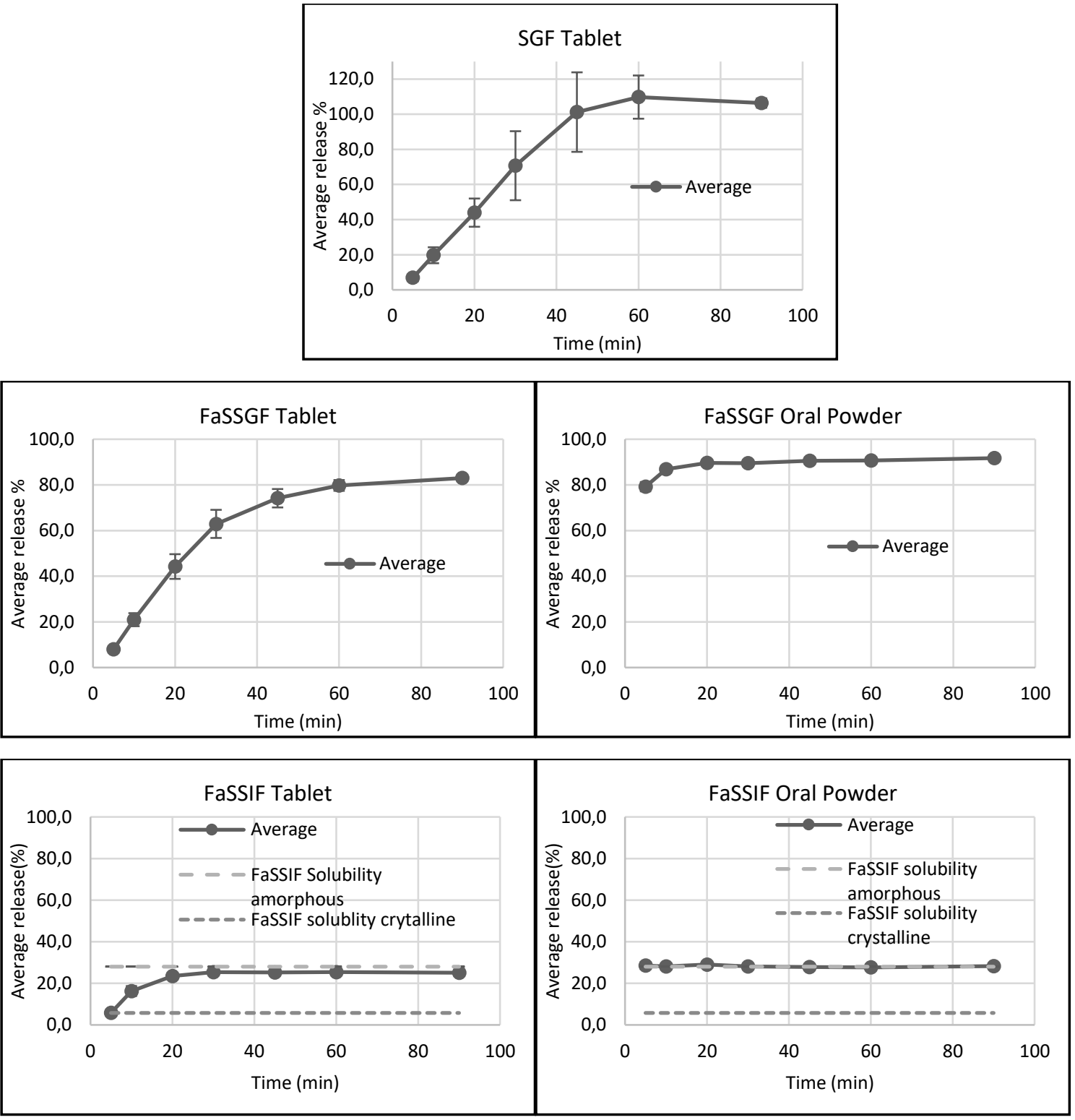

Fig. 6 Mean \pm SD $(n=6)$ dissolution profiles of Norvir $^{\circledR}$ formulations expressed as \% release (standard deviations are smaller than symbols at most time points) 


\subsection{Two-stage experiments}

To investigate the behavior of Norvir ${ }^{\circledast}$ tablets and powder during and after passage from the stomach to the small intestine, two-stage experiments were performed. As observed in single-stage dissolution, the use of compendial media leads to an over-prediction of the maximum drug release in the gastric compartment. For the oral powder formulation complete drug release was observed in the compendial medium within 30 minutes, while in biorelevant media the release after 30 minutes was $90 \%$. For the tablet formulation drug release was not complete at the 30 minute media transfer time point, which is also in agreement with the results from the single-stage experiments. Final drug concentrations in the resulting media representing the intestinal phase were underestimated when using SIF media ( $30 \mu \mathrm{g} / \mathrm{ml}$ ), while the final concentration in FaSSIF-V1 was $56 \mu \mathrm{g} / \mathrm{ml}$, which was in good agreement with the reported amorphous solubility.

For the intestinal phase, experiments were terminated after 120 minutes since a plateau had been reached.

As with the single-stage dissolution experiments, the results of the two-stage tests were also mechanistically modeled using the SIVA toolkit. Since precipitation to the amorphous solubility was observed to be very fast after media transfer, the critical supersaturation ratio (CSR) was set at 1 and the secondary precipitation rate constant was set to $1000 \mathrm{~h}^{-1}$, the maximum value possible. The primary precipitation rate was set as $0.0001 \mathrm{~h}^{-1}$, since no relevant further precipitation to the crystalline thermodynamic solubility was observed. More detail about the empirical precipitation model used in this model is further described by Pathak et al. ${ }^{13}$.
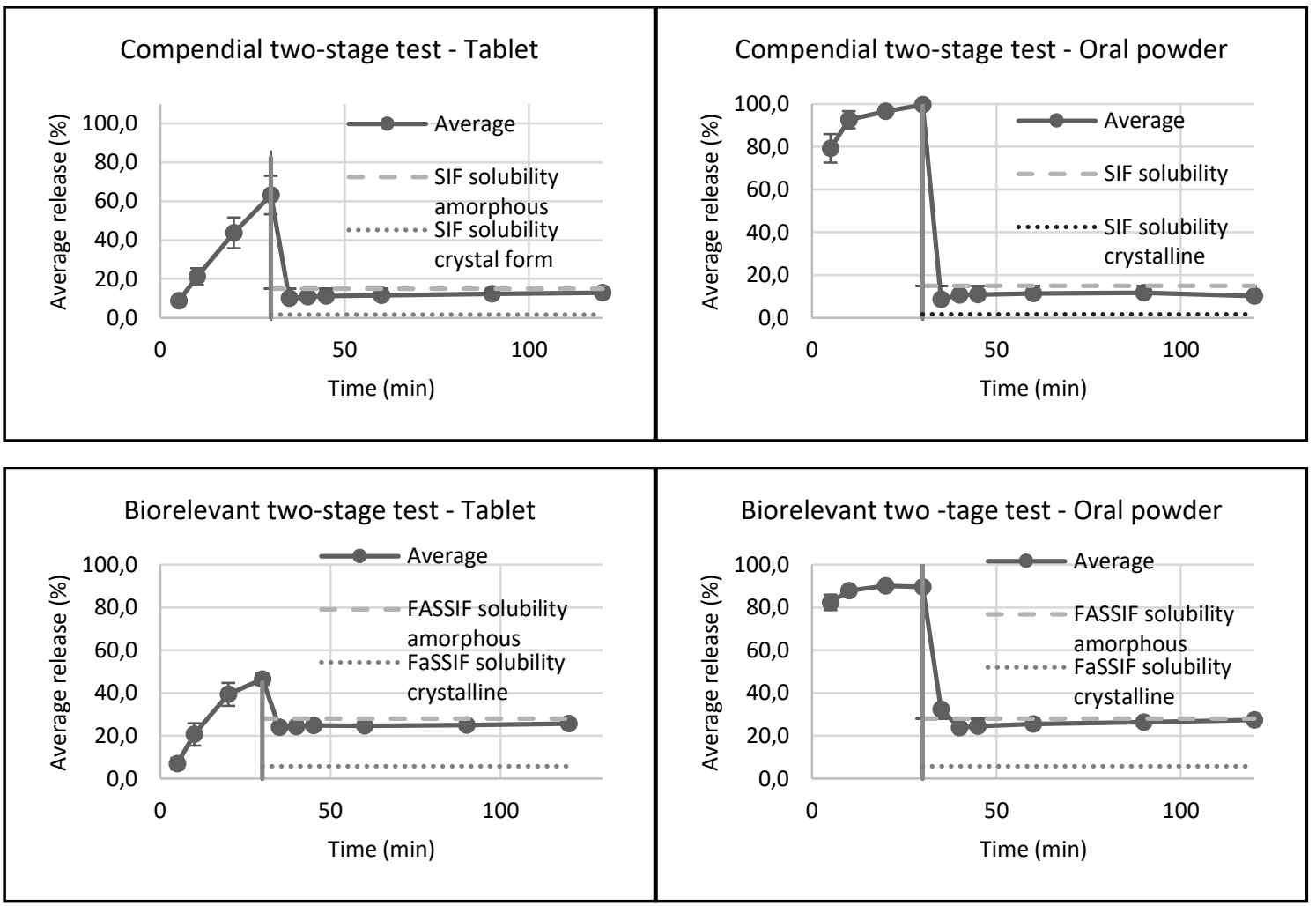

Fig. 7 Mean \pm SD $(n=6)$ two-stage test profiles of Norvir ${ }^{\circledR} 100 \mathrm{mg}$ tablets and oral powder expressed as $\%$ release. The top panels show data in compendial buffers, while the bottom panels show data in biorelevant media (standard deviations are smaller than symbols at most time points) 


\subsection{Transfer experiments}

In transfer experiments, concentrations higher than amorphous solubility of ritonavir in the intestine simulating medium for either Norvir ${ }^{\circledR} 100$ mg tablets or powder were not observed (Figure 6). Concentrations approached the apparent amorphous solubility by 30 minutes but this concentration was never exceeded. It is assumed that ritonavir which had been dissolved in the gastric test compartment immediately precipitated to its amorphous solubility after arriving in the intestinal compartment. Thereafter, ritonavir concentrations remained at the apparent amorphous solubility over the 3 hours of the experiment.
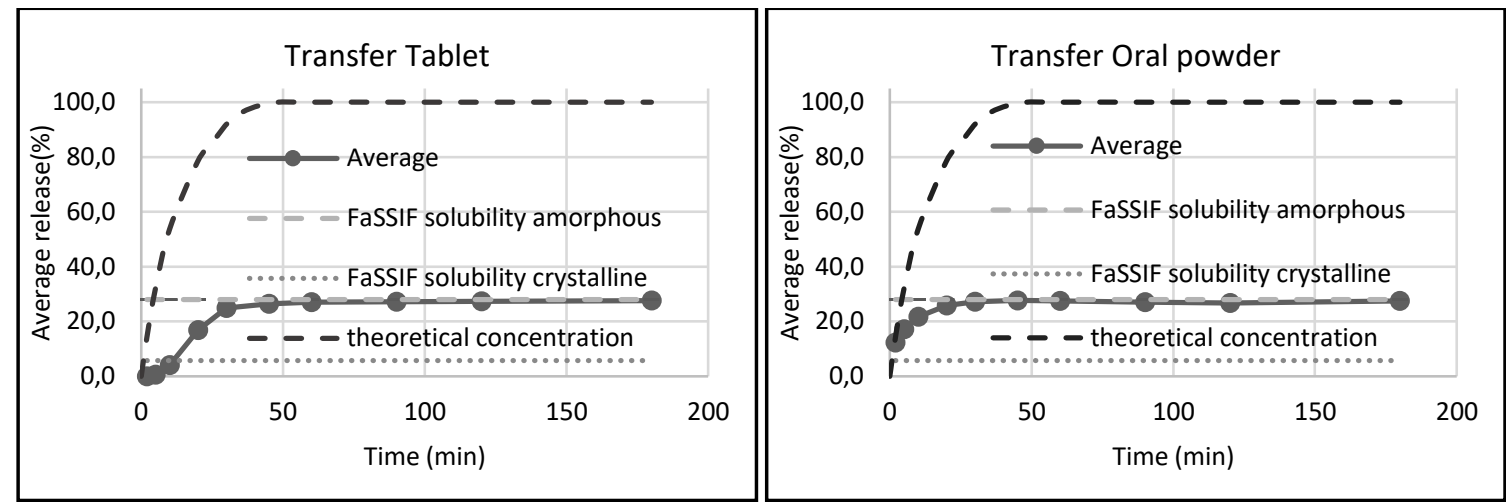

Fig. 8 Mean \pm SD $(n=3)$ transfer profiles expressed in \% release

Although ritonavir is a weakly basic drug, and concentrations higher than the amorphous solubility might have been expected to occur temporarily upon transfer (vis à vis the two-stage test), no differences with respect to the supersaturation and precipitation of the Norvir ${ }^{\circledast} 100 \mathrm{mg}$ formulations were observed in the more complex transfer experiments compared with the simpler two-stage experiments.

\subsection{Refined Developability Classification System (rDCS)}

Ritonavir is a weakly basic drug with two pKa values (1.9 and 2.6) and has been reported in the literature to be a BCS class IV drug ${ }^{36,37}$. The usual clinical dose is $100 \mathrm{mg}$ twice daily when used as a pharmacokinetic booster ${ }^{38}$. Rather than using simple buffers systems to determine drug solubility as in the BCS, the rDCS proposes the use of FaHIF (fasted human intestinal fluid) solubility as a benchmark to achieve a more accurate classification with respect to in vivo drug solubility. When no FaHIF data are available, the rDCS standard data set of FaHIF and FaSSIF V1 data for 60 compounds $\left(r^{2}=0.84\right)$ can be used to correlate the solubility in FaSSIF to a value in FaHIF. In its crystalline form, the solubility of ritonavir in FaSSIF-V1 was determined to be $11.5 \mu \mathrm{g} / \mathrm{ml}$. For ritonavir a FaHIF value of $15 \mu \mathrm{g} / \mathrm{ml}$ was calculated on this basis from the correlation. Comparing this estimate with two FaHIF solubilities for crystalline ritonavir reported in the literature, $4.8 \mu \mathrm{g} / \mathrm{mL}^{39}$ and $34.6 \mu \mathrm{g} / \mathrm{mL},{ }^{40}$ indicates that the rDCS approach is in line with direct measurement in FaHIF.

Similarly, for the rDCS permeability classification, in-house permeability data from Caco 2 cell line experiments can be correlated with an rDCS standard data set to calculate $P_{\text {eff }}$ values if these are not available for the drug in question. Since neither $P_{\text {eff }}$ data nor Caco 2 data was available for ritonavir, 
$P_{\text {eff }}$ was calculated in the Simcyp Simulator using the MechP $P_{\text {eff }}$ model to be $7.25 \times 10^{-4} \mathrm{~cm} / \mathrm{s}^{2}$ for the jejunum. Based on calculated FaHIF solubility and $P_{\text {eff }}$ permeability data, ritonavir was consequently classified according to the rDCS as follows.

First, the "Absorption number" was calculated according to Eq. 1. In this equation, DF is the degree of flatness with a standard value of $1.7, \mathrm{P}_{\text {eff }}$ is the effective small intestinal permeability, $\mathrm{T}_{\mathrm{si}}$ is the small intestinal transit time set at 3.32 hours (for calculations this value is adjusted to seconds) and $R_{\mathrm{si}}$ is the radius of the gut, set at $2 \mathrm{~cm}$. The "Absorption number" for ritonavir was calculated to be 7.37, which classifies ritonavir as an easily absorbable drug, either rDCS class I or II.

$$
A n=\frac{D F x P_{e f f(s i)} x T_{S i}}{R_{S i}}
$$

Equation 1

Next, the "Dose number" was calculated according to Eq. 2. As previously mentioned, the dose of ritonavir is $100 \mathrm{mg}$, while $\mathrm{S}_{\mathrm{si}}$ is the solubility of the drug in the small intestine (FaHIF in $\mathrm{mg} / \mathrm{ml}$ )) and $V_{\mathrm{si}}$ is the effective available fluid volume for dissolution in the small intestine $(500 \mathrm{~mL})$. The "Dose number" for ritonavir was calculated to be 10.15. Combination of the "Absorption number" and "Dose number" classifies ritonavir as an rDCS class II drug.

$$
D o=\frac{\text { dose }}{S_{S i} \times V_{S i}}
$$

Equation 2

To define the boundary between rDCS class Ila and IIb, the SLAD (solubility limiting absorbable dose) is calculated. In Eq. 3, $\mathrm{V}_{\mathrm{si}}$ is the effective volume of the fluid in the small intestine available for drug dissolution $(500 \mathrm{~mL}$ ). For ritonavir, an orally administered dose of $72.59 \mathrm{mg}$ is the resulting boundary between class Ila and IIb.

$$
S L A D=S_{S i} \times V_{S i} \times A n
$$

Equation 3

At a $100 \mathrm{mg}$ dose, the SLAD limit of $72.59 \mathrm{mg}$ is exceeded, hence ritonavir is classified as an rDCS class Ilb drug. For drugs falling in this classification, an enabling formulation is usually needed to overcome the solubility limitation.

Norvir ${ }^{\circledR}$ tablets and oral powders are formulated as amorphous solid dispersions (ASD), which is one of the various enabling options for oral formulation ${ }^{23,41}$. For Norvir ${ }^{\circledR}$ formulations an improved FaHIF solubility can be calculated from the solubility of the ritonavir amorphous formulations in FaSSIF (60 $\mu \mathrm{g} / \mathrm{ml})$. Using the rDCS standard data set, the FaHIF solubility of the amorphous form was calculated to be $62 \mu \mathrm{g} / \mathrm{ml}$. As a result, a new "Dose number" of 3.22 was calculated for Norvir ${ }^{\circledast} 100 \mathrm{mg}$ ASD formulations. Although this value still corresponds to rDCS class II, the SLAD value increases to 228.47 $\mathrm{mg}$, thereby shifting the $100 \mathrm{mg}$ Norvir $^{\circledR}$ formulations into the more favorable rDCS class Ila region, with only dissolution rate limitations to absorption.

Additionally, for rDCS classes I, Ila and III it is suggested to calculate a target maximum particle size, below which dissolution will no longer be rate limiting to drug absorption. For this purpose, Eq. 4 was applied, where $r$ represents the target particle radius, $D$ is the diffusion coefficient $\left(5 \times 10^{-6} \mathrm{~cm}^{2} / \mathrm{s}\right), D n$ is the "Dissolution number" (set as 1 ) and $\rho$ is the drug density $\left(1.2 \mathrm{~g} / \mathrm{cm}^{3}\right)$. For Norvir ${ }^{\boxplus}$ formulations, a target radius of $30.4 \mu \mathrm{m}$ was calculated. 


$$
r^{2}=\frac{3 D \times S_{S i} x T_{S i}}{D n x \rho}
$$

Interestingly, for most drug formulations a default particle size of $10 \mu \mathrm{m}$ is applied in PBPK modelling software Simcyp Simulator - perhaps in anticipation that many drugs will be micronized to improve their dissolution rate. At this particle size, dissolution should not be rate limiting for ritonavir absorption from the ASD formulation.

However, since weak bases are prone to supersaturation and precipitation in the small intestine after gastric emptying and prior dissolution in the stomach, an rDCS classification based solely on the above-described and applied "standard investigations" might lead to poor prediction of actual in vivo performance, since these supersaturation/precipitation phenomena are not captured by FaHIF solubility data alone. Determination of drug solubility in gastric and intestinal media represent a physiological "best and worst case" scenario for weakly basic drug compounds, since solubility will be the highest in the acidic fasted stomach and the lowest in the more basic duodenal lumen. However, single-stage dissolution experiments in separate media may fail to provide a representation of actual in vivo drug behavior upon gastric emptying into the duodenum for weakly basic drugs. Therefore, further "customized investigations" in the form of biorelevant two-stage experiments that mimic gastric dissolution and subsequent gastric emptying into the small intestine the behavior are triggered for poorly soluble weak bases by the $\mathrm{rDCS}^{6}$. In case of the Norvir ${ }^{\circledast}$ formulations, immediate precipitation to the amorphous drug solubility is apparent after media transfer in both the two-stage and transfer experiments. In general, two-stage experiments will detect whether or not the drug formulation will supersaturate but then precipitate, but for highly supersaturating and slowly precipitating drugs, the transfer model may be more accurate to determine the kinetics of the precipitation behavior and hence the concentration of drug available in the small intestine for absorption. In the case of both Norvir ${ }^{\circledR}$ formulations, concentrations exceeding the amorphous solubility of the ASD were not detected in either two-stage or transfer experiments.

\subsection{Prediction of intraluminal duodenal drug behavior with biorelevant in vitro dissolution experiments and PBPK modelling}

As observed in the previously described two-stage and transfer experiments, ritonavir precipitates almost immediately to its amorphous form after media transfer. Since bile salt concentrations in the human intestinal tract are fluctuating and highly variable, the use of conventional FaSSIF media with a bile salt concentration of $3 \mathrm{mMol}$ and $0.75 \mathrm{mMol}$ of lecithin to determine actual intestinal drug behavior can be viewed as an approach to determine the average in vivo behavior. Minimum and maximum reported values of small intestinal luminal bile salt concentrations in the literature have been reported to be between $0.3 \mathrm{mMol}$ and $9 \mathrm{mMol}{ }^{42}$. For this reason, dissolution experiments at different bile salt concentrations with attendant changes in lecithin concentrations were conducted for the Norvir ${ }^{\circledR}$ oral powder formulation to determine the impact of different bile component concentrations on the amorphous solubility of ritonavir formulated as an ASD. Results in Figure 7 showed that for higher bile salt and lecithin concentrations the amorphous solubility of ritonavir increased, while for media without any bile salt components solubility decreased to less than the half of the solubility observed in the standard FaSSIF-V1 composition. For FaSSIF media with double concentrated bile concentrations, the amorphous solubility increased by a factor of 1.25 to around $70 \mu \mathrm{g} / \mathrm{ml}$. 


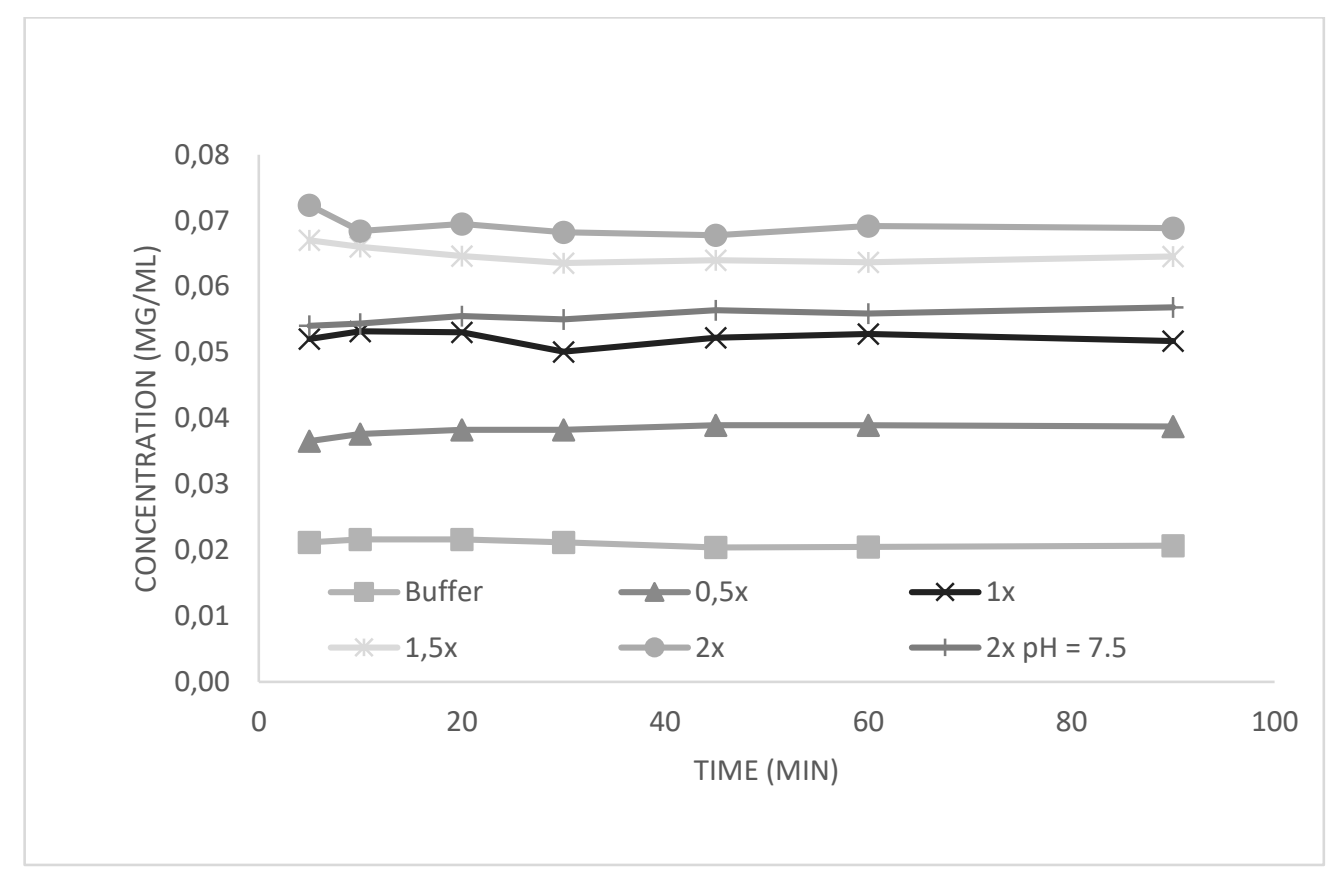

Fig. 9 One stage dissolution results of $\mathrm{Norvir}^{\circledast}$ powder in FaSSIF at different bile salt concentrations

Intraluminal drug concentrations measured in samples taken from the duodenum of 5 healthy volunteers are shown in Figure 8a. Measured amorphous FaSSIF solubility at different physiologically relevant bile salt concentrations were able to capture the in vivo intraluminal ritonavir concentrations of the data set from the 5 study subjects with respect to their maximum measured concentrations over a time of 2 hours. Results show an average maximum dissolved drug concentration of $47 \mu \mathrm{g} / \mathrm{ml}$ at 40 minutes, which is in line with the solubility of the amorphous compound in half-concentrated bile salt containing FaSSIF-V1. In healthy volunteer Subjects 3 and 4 , the maximum observed intraluminal concentrations slightly exceeded the in vitro concentration of the Norvir ${ }^{\circledast}$ oral powder in double bile salt concentrated FaSSIF media, while Subject 1 reached a maximum concentration rather late, 75 minutes after administration, and this concentration was just above the solubility in the FaSSIF buffer (containing no bile components). 
(a)

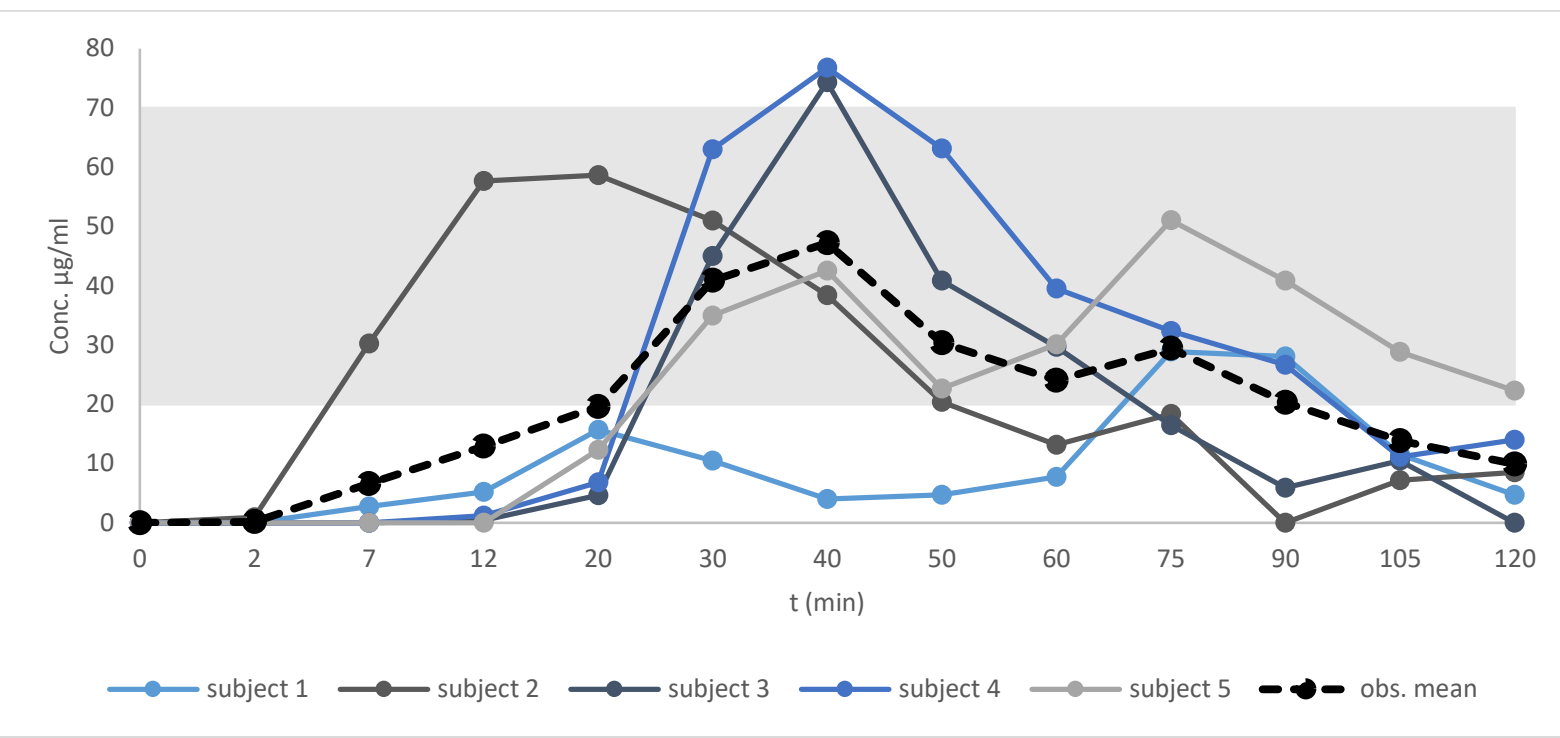

(b)

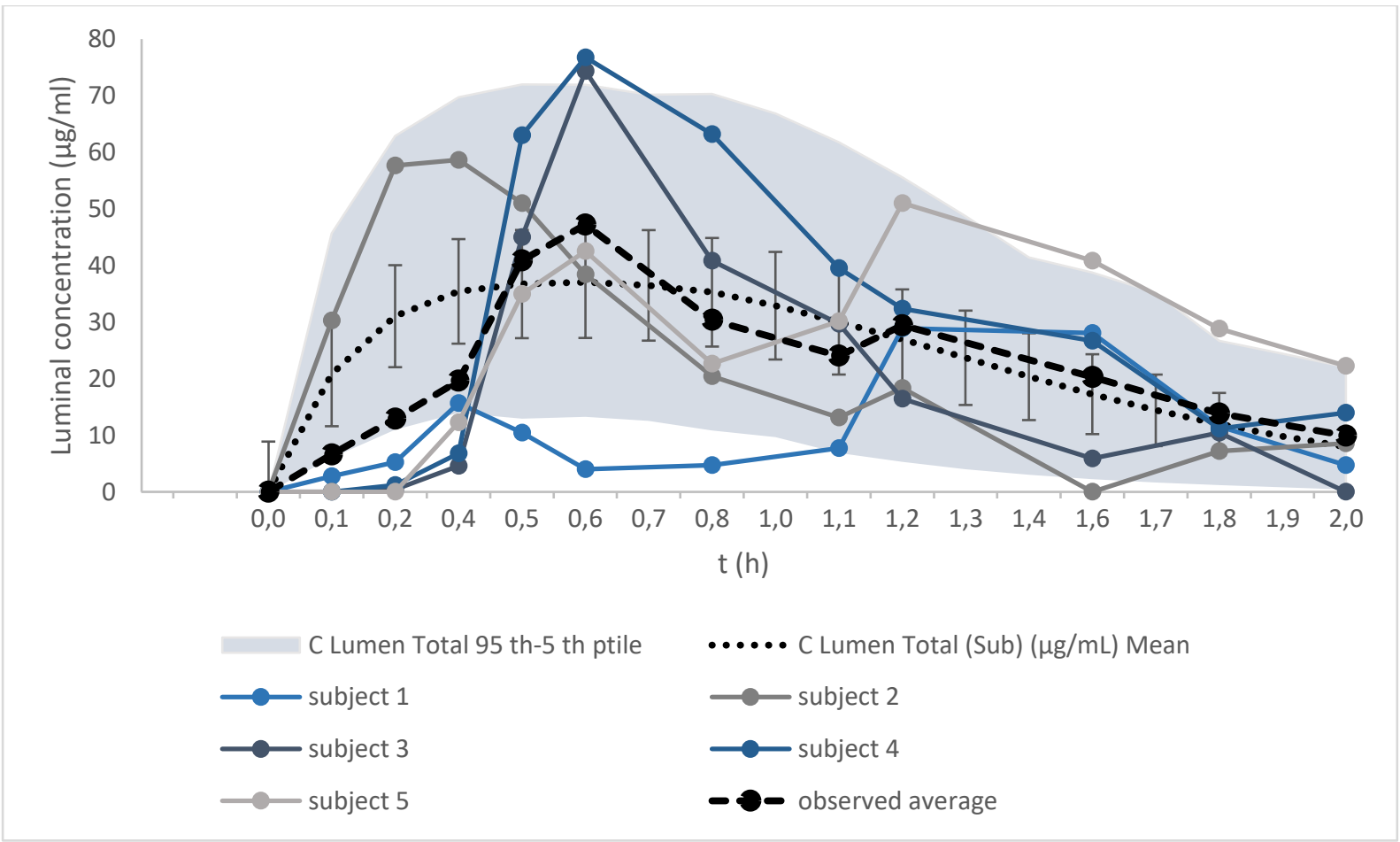

Fig. 10 Observed intraluminal ritonavir concentration for 5 subjects compared with a) measured in vitro ritonavir concentrations between FaSSIF versions containing bile salt concentrations ranging from 0 to $6 \mathrm{mM}$ (with attendant changes in the concentration of lecithin) shown as the grey shaded area and b) results from 10 simulated trials with standard deviations (---), with the grey background representing the $95 \% \mathrm{Cl}$ range.

The option to predict intraluminal drug concentrations at different parts of the gastrointestinal tract after drug intake is available in the Simcyp Simulator ADAM model. Taking into consideration that precipitation to the crystalline form of ritonavir did not occur in the in vitro biorelevant two-stage dissolution experiments, precipitation parameters were adjusted accordingly and built into the model using a CSR (critical supersaturation ratio) value of 1 (no supersaturation expressed as a ratio 
of 1 between highest concentrations observed in the intestinal test stage and the amorphous solubility) and a PRC (precipitation rate constant) value $0.0001 \mathrm{~h}^{-1}$ (expressing negligible precipitation to the crystalline form). Results are shown in Figure $8 \mathrm{~b}$. In the simulated trials, a maximum ritonavir concentration of $37 \mu \mathrm{g} / \mathrm{ml}$ was reached shortly after 30 minutes, which is very close to the average $t_{\max }$ value in the mean profile of the clinical data (40 minutes). The simulated average maximum concentration $37 \mu \mathrm{g} / \mathrm{ml}$ corresponds to the in vitro measured amorphous solubility of ritonavir in FaSSIF with $0.5 x$ bile salt and lecithin contents. In the individual simulations, the maximum ritonavir concentration in the duodenum was simulated to be $79 \mu \mathrm{g} / \mathrm{ml}$ (subject with $7.6 \mathrm{mM}$ duodenal bile salt concentration) and the lowest maximum luminal ritonavir concentration was simulated to be 17 $\mu \mathrm{g} / \mathrm{ml}$ (subject with a $1.3 \mathrm{mM}$ duodenal bile salt concentration). Observation and simulation of concentrations lower than the amorphous solubility of ritonavir in the ASD $(20 \mu \mathrm{g} / \mathrm{mL}$ and above, depending on bile salt level) can be likely attributed to absorption of the drug as it dissolves.

Taken together, the in vitro and in vivo results suggest that in vivo luminal drug concentrations in the duodenum after oral intake of $\mathrm{Norvir}^{\circledR}$ formulations can be predicted adequately with in vitro dissolution experiments using biorelevant media adjusted to different physiological bile salt concentrations, while PBPK modelling provided an excellent simulation of the mean results and captured essentially the whole range of measured luminal drug concentrations.

\subsection{PBPK model simulations of plasma concentrations}

First, the original PBPK model proposed by Arora et al. was used to simulate the data of $\mathrm{Ng}$ et al. to check user-to-user reproducibility. Simulations were identical to those reported by Arora et al. (data not shown). Then, the PBPK model adapted from Arora et al. (see parameters in Table 2) to simulate ritonavir luminal concentrations, was used to simulate the plasma profiles from $\mathrm{Ng}$ et al. to verify the accuracy of the adapted PBPK model. As a third step, the adapted model was used to simulate the plasma profiles observed in the clinical trial at KU Leuven.

\subsubsection{Verification of in-house PBPK model against literature data from $\mathrm{Ng}$ et al.}

For verification of the model adapted from Arora et al., simulated profiles were compared with literature data in 27 healthy volunteers from $\mathrm{Ng}$ et al. As shown in Figure 9, the adapted model (10 simulated trials, each with 27 subjects) shows a very good fit with the observed data. 


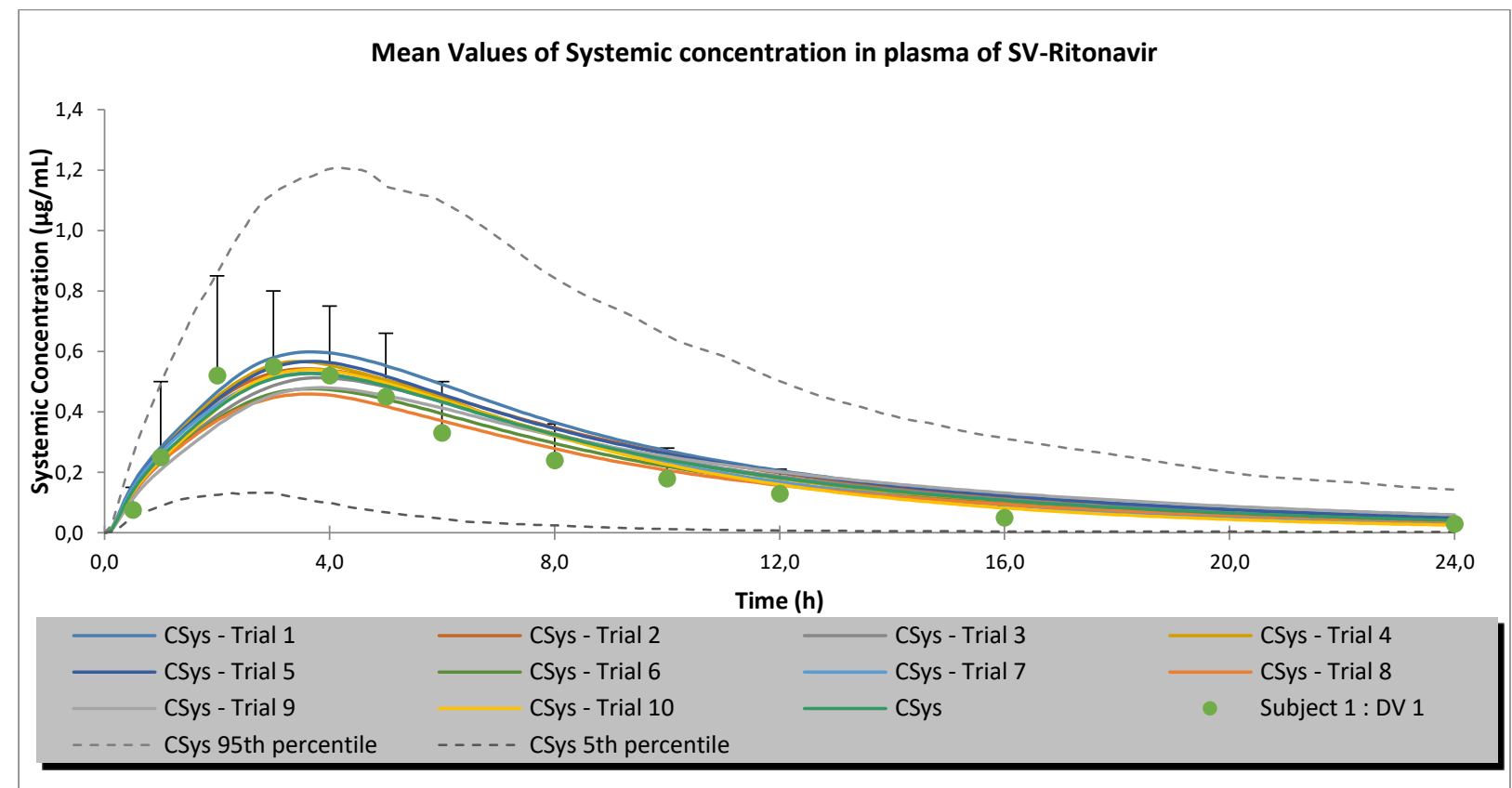

Fig. 11 Simulated mean plasma profiles of 10 trials, each with 27 subjects, shown with standard deviations and $5^{\text {th }}$ to $95^{\text {th }}$ percentile range for the grand mean profile.

The simulated profiles from 10 trials show only minor differences regarding average $C_{\max }, t_{\max }$ and $\mathrm{AUC}_{0-\mathrm{t}}$ values compared with the observed data set by $\mathrm{Ng}$ et al.. The high standard deviations of the observed data suggest complex inter-individual variability in the pharmacokinetics of ritonavir. The standard deviations were captured easily by the confidence interval between the $5^{\text {th }}$ and $95^{\text {th }}$ percentile in the simulations. Interestingly, the $t_{\max }$ and $\mathrm{AUC}_{0-\mathrm{t}}$ parameters were captured slightly better by the initial model by Arora et al., while the $C_{\max }$ value is more captured better with the adapted model (see table 4). However, all PK parameters were within a 0.8-1.25 fold range and AAFE was well below a value of two for both models, which is generally regarded as a successful simulation.

Table 4

Calculated fold error, average fold error and absolute average fold error for ritonavir simulations against observed data from Ng et al.

\begin{tabular}{llll}
\hline PBPK model & $\mathrm{FE}\left(\mathrm{C}_{\max } / \mathrm{AUC}_{0-\mathrm{-}} / \mathrm{t}_{\mathrm{max}}\right)$ & AFE & AAFE \\
\hline $\begin{array}{l}\text { Arora et al. } \\
\begin{array}{l}\text { Adapted model } \\
\text { (in vitro data applied) }\end{array}\end{array}$ & $0.82 / 0.97 / 1.07$ & 0.95 & 1.11 \\
$\begin{array}{l}\text { Adapted model } \\
\text { (SIVA calculation of } \\
\text { biorelevant solubility*) }\end{array}$ & $1.33 / 1.14 / 1.08$ & 1.05 & 1.09 \\
\hline
\end{tabular}

*to obtain this value, the final concentration observed in single stage testing using compendial (buffer) media was used to calculate the equivalent biorelevant solubility with the Simcyp "Predictions toolbox"

To emphasize the importance of obtaining input parameters using appropriate in vitro experiments, two very different simulations are shown in Figure 10. First, simulations for the Norvir ${ }^{\circledR} 100 \mathrm{mg}$ tablet 
formulation based purely on the crystalline form of ritonavir would drastically under-predict the in vivo bioavailability of the formulations (Figure 10a). In the second case, solubility and dissolution experiments were conducted in compendial buffer media, then the biorelevant solubility and the LogK $\mathrm{K}_{\mathrm{m}: \mathrm{w}}$ were predicted based on QSAR (quantitative structure-activity relationship) and $\mathrm{pH}$ dependent solubility in the Simcyp Simulator. Even though simulations still predict the plasma profile reasonably well with an AFE value of 1.22 and AAFE of 1.35, the observed plasma concentrations tend to be over-predicted using this approach (Figure 10b). Only when applying solubility and dissolution experiments in biorelevant media (FaSSGF and FaSSIF) with the formulated solid state of the dosage form were quantitatively accurate simulations obtained (Figure 9).

(a)

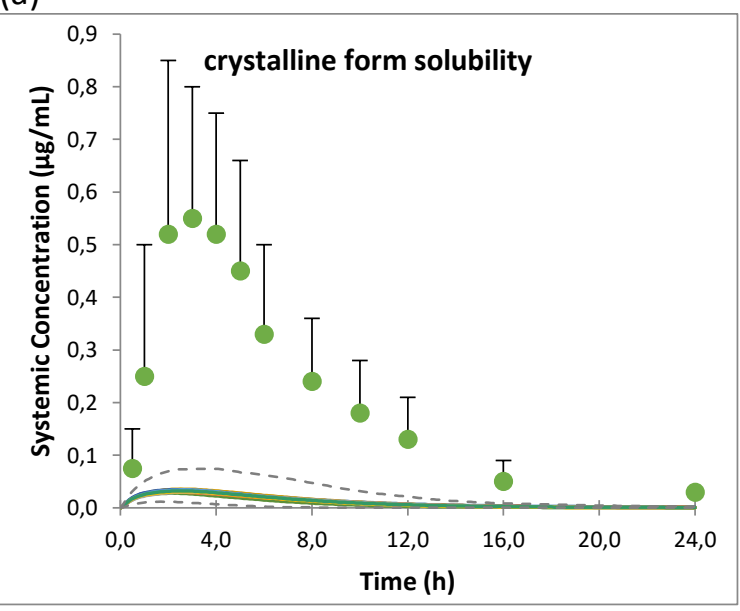

(b)

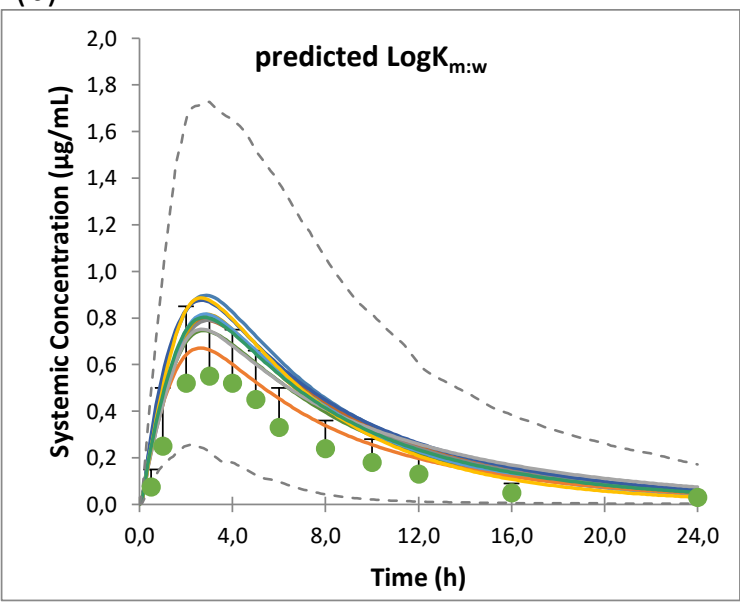

Fig. 12 Simulated plasma profiles from 10 trials, each with 27 subjects, based on a) crystalline solubility data of ritonavir and b) amorphous biorelevant solubility obtained from the final concentrations in the single-stage tests in compendial media, together with the mean observed plasma profile of 27 subjects with standard deviation and 5 th to 95 th percentile.

\subsection{2. $\quad$ Comparison of simulations using the adapted model with plasma concentration data from Leuven}

The adapted model was further compared with the plasma concentration data from the same KU Leuven in which the intraluminal drug concentrations were measured. Results are shown in figure 11. Unfortunately, the data set of the 5 healthy volunteer study shows a very high standard deviation at all sampling time points, which is partly attributable to limited number of study participants. Furthermore, the elimination behavior was not fully characterized and $\mathrm{AUC}_{0-\mathrm{t}}$ could not be determined from the study results, since sampling was truncated after 8 hours. The overall shape of the observed profile failed to fit the simulated trials compared to the simulated profiles and the overall profile. However, drug concentrations in the simulated profiles all fell within the high standard deviation of the observed concentrations of study results as well as the average $C_{\max }$ and $t_{\max }$ values over the first five hours. The $C_{\max }$ and $t_{\max }$ values averaged over the virtual trials also captured the observed values well. 


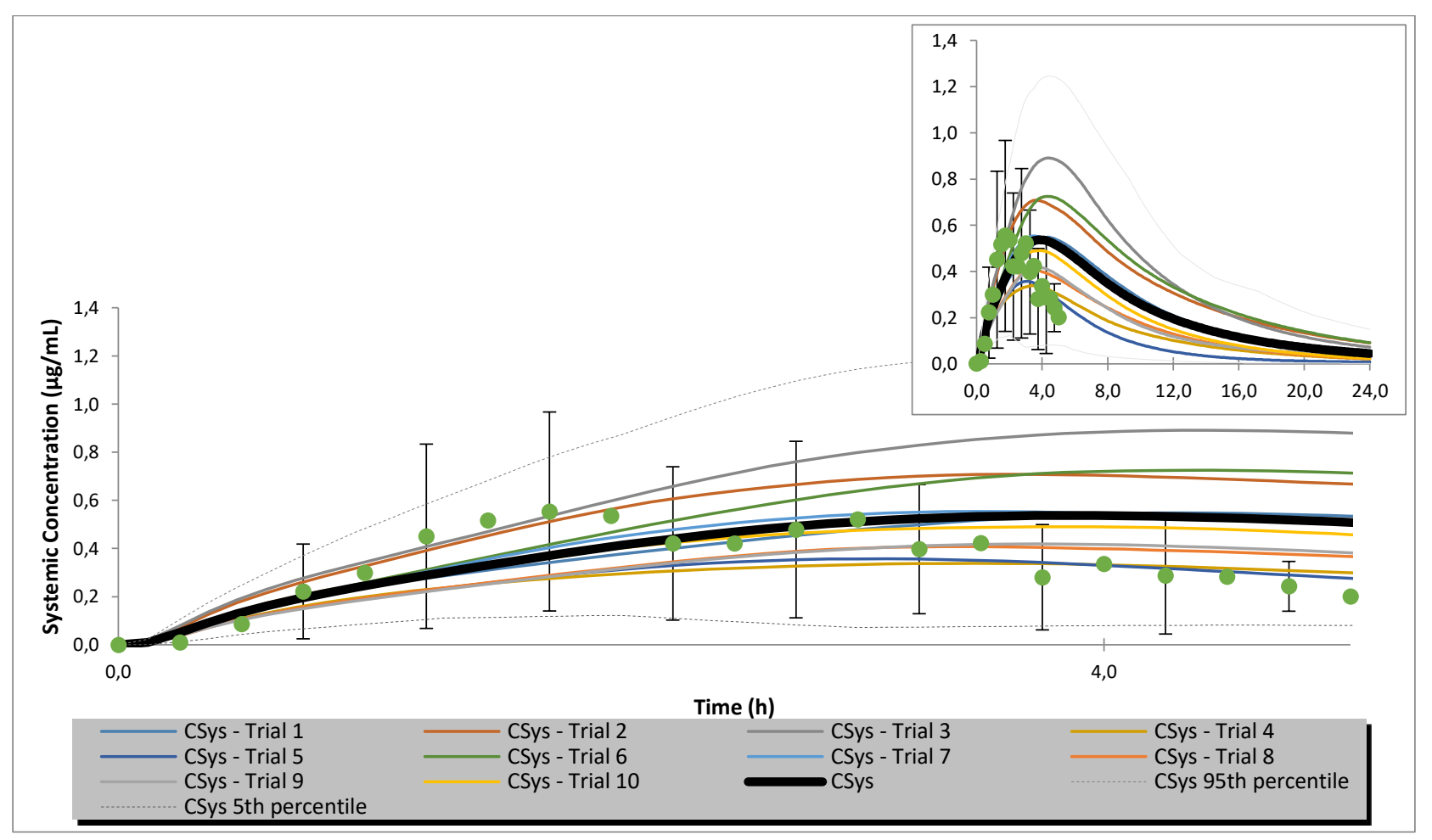

Fig. 13 Simulations of ritonavir pharmacokinetics after p.o. administration (10 trials with 5 subjects each) and mean observed in vivo data of 5 subjects (green dots) with standard deviation and $5^{\text {th }}$ to $95^{\text {th }}$ percentile

The simulated $t_{\max }$ was calculated to be 1.4 fold of the observed $t_{\max }$ value and the simulated $C_{\max }$ was within the 0.8-1.25 fold range with 0.95. Calculations of AFE and AAFE were again well below 2 with 1.15 and 1.21 for five the first hours.

Table 5

Comparison of PK parameters for observed and PBPK simulated data from the KU Leuven study

\begin{tabular}{llll|ll}
\hline PK Parameters & $\begin{array}{c}\text { Observed } \\
\text { 27 subjects } \\
\text { (Ng et al.) }\end{array}$ & $\begin{array}{c}\text { Initial model } \\
\text { 10 trials, each } \\
\text { 27 subjects } \\
\text { (Arora et al.) }\end{array}$ & $\begin{array}{c}\text { adapted model } \\
\text { 10 trials, each } \\
\text { 27 subjects }\end{array}$ & $\begin{array}{c}\text { Observed } \\
\text { Leuven (5 } \\
\text { subjects) }\end{array}$ & $\begin{array}{c}\text { adapted model } \\
\text { 10 trials, each 5 } \\
\text { subjects }\end{array}$ \\
\hline$C_{\max }(\mu \mathrm{g} / \mathrm{ml})$ & $0.6 \pm 0.3$ & $0.49 \pm 0.3$ & $0.57+0.34$ & $0.61 \pm 0.45$ & $0.58+0.36$ \\
$t_{\max }(h)$ & $3.2 \pm 1.2$ & $3.43 \pm 1.18$ & $3.45+1.06$ & $2.6 \pm 0.94$ & $3.64+1.16$ \\
$A \cup C_{0-t}(\mu \mathrm{g} / \mathrm{ml} . \mathrm{h})$ & $4.6 \pm 2.0$ & $4.46 \pm 3.27$ & $5.26+3.82$ & n.d. & $5.51+4.11$ \\
\hline
\end{tabular}

Although the Leuven data set showed a very high standard deviation among the 5 volunteers, $C_{\max }$ and $t_{\max }$ still fell within the standard deviations of the $\mathrm{Ng}$ et al. study. It appears that the clearance of the study participants was faster for the subjects in the KU Leuven study than for the participants of the Ng et al. trial i.e. that different elimination kinetics in the Leuven cohort are the root cause of the discrepancies between the simulated and observed profiles at longer times. 


\subsection{Norvir ${ }^{\circledR}$ oral powder formulation}

As shown in in vitro single and two-stage dissolution experiments tests, differences in dissolution between the Norvir ${ }^{\circledR} 100 \mathrm{mg}$ oral powder and the tablet formulation were modest. This can be partly explained by the fact that disintegration of $\mathrm{Norvir}^{\circledR}$ tablets was shown to be already very fast in in vitro dissolution experiments. In vivo, the plasma profiles reported by Salem et al. after administration of the Norvir ${ }^{\circledR} 100 \mathrm{mg}$ oral powder formulation in 24 subjects indicated a slightly higher mean $\mathrm{C}_{\max }$ value of $0.72 \mu \mathrm{g} / \mathrm{ml}$ for the $100 \mathrm{mg}$ oral powder compared to the Norvir ${ }^{\circledR}$ tablet formulations ${ }^{18}$. The simulations of the oral powder formulation attained by changing the DLM scalar value to 1 (representing immediate drug dissolution) tended to be somewhat lower than the mean profile observed in the clinical study. However, since very high standard deviations $(0.72 \pm 0.4 \mu \mathrm{g} / \mathrm{mL})$ were reported, the simulations fell well within the observed concentration ranges.

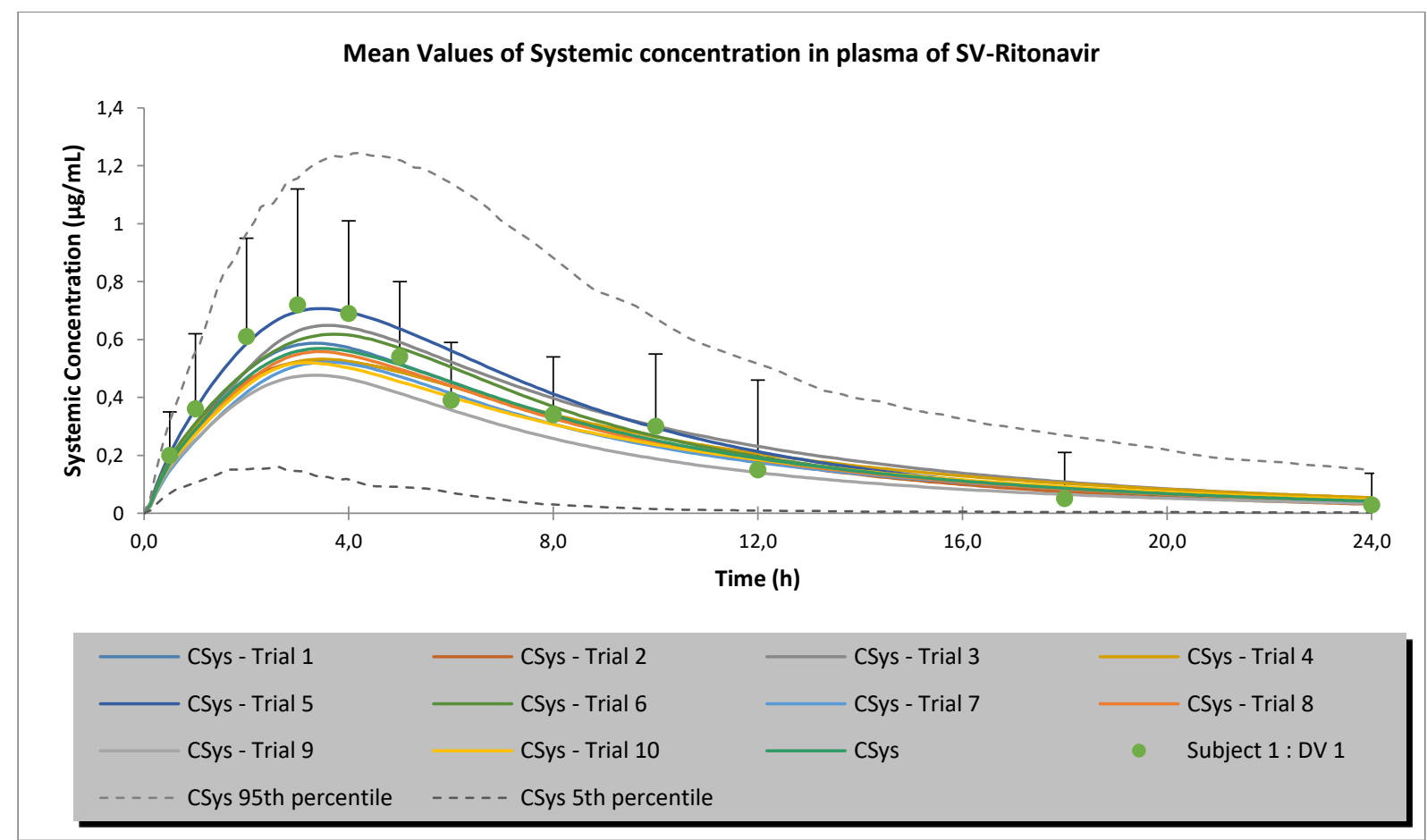

Fig. 14 Simulated plasma profiles from 10 trials, each with 24 subjects, and the grand mean observed plasma profile from 24 subjects reported by Salem et al., along with standard deviations and $5^{\text {th }}$ to $95^{\text {th }}$ percentile ranges

Table 6

Comparison of PK parameters for observed and PBPK modeled data

\begin{tabular}{llll}
\hline PBPK model & FE $\left(\mathrm{C}_{\max } / \mathrm{AUC}_{0-\mathrm{t}} / \mathrm{t}_{\max }\right)$ & AFE & AAFE \\
\hline adapted model & $0.79 / 0.88 / 1.13$ & 0.92 & 1.18 \\
\hline
\end{tabular}




\section{Discussion}

In this study, it was shown that by following rDCS in vitro solubility and dissolution experiments, the weakly basic drug ritonavir and its enabling ASD formulations could be classified into class IIb and Ila, respectively. Using this approach, the pharmaceutical scientist is able to determine the level of formulation challenge for a weakly basic drug and the extent to which an enabling formulation can overcome this challenge.

Results of solubility and single and two-stage dissolution in vitro experiments of varying complexity were coupled with in silico PBPK modelling programs (SIVA \& Simcyp Simulator). By applying biorelevant dissolution tests with intestinal media at different bile salt and lecithin concentrations in the physiological ranges, it was possible to predict the intraluminal drug concentrations observed after administration of the ritonavir $\mathrm{Norvir}^{\circledR}$ tablet formulation. Therefore, it seems reasonable to apply different variations of the simulated intestinal media to cover the "best" and "worst case" scenarios in vivo in addition to using the standard FaSSIF version to represent the average intestinal fluid, when in vitro luminal concentrations are to be predicted.

For ritonavir formulations, the transfer model was not able to give any further insight on the supersaturating and precipitation behavior of the Norvir ${ }^{\circledR}$ formulations compared to the simpler twostage experiments, so the latter results were used for parameter input into PBPK modelling to successfully predict intraluminal and plasma concentrations.

Weakly basic drugs supersaturate and precipitate to varying degrees when entering the small intestine due to the steep change in the $\mathrm{pH}$ value. Since supersaturation is the driving force for intestinal drug absorption, enabling formulations are often sought for weakly basic drugs to increase the degree of supersaturation and prolong this thermodynamically unstable state. However, the effectiveness of the formulation effort varies greatly among drug products. For example, the enabling formulation of etravirine (Intellence ${ }^{\circledR}$ ) was shown to precipitate quite slowly to the crystalline solubility during transfer experiments, while Norvir ${ }^{\circledR}$ ritonavir formulations precipitated immediately to the solubility of the amorphous form, but did not revert to the crystalline solubility during the experimental time-frame ${ }^{43}$.

These different behaviors might be partly due to the inherent precipitation and supersaturation properties of the drug, as exemplified by ketoconazole (Nizoral), cinnarizine (Arlevert ${ }^{\circledast}$ ) or atazanavir $\left(\text { Reyataz }{ }^{\circledR}\right)^{15,44,45}$. While for ketoconazole and cinnarizine sensitivity of the precipitation rate to different in vitro simulated gastric emptying patterns was reported, atazanavir (like ritonavir) showed no differences between transfer and two-stage experiments. In the context of physiology, transfer model experiments can be used to represent a wide range of gastric emptying patterns, while media transfer by immediate pouring (the two-stage test) represents a "worst case" scenario for drug

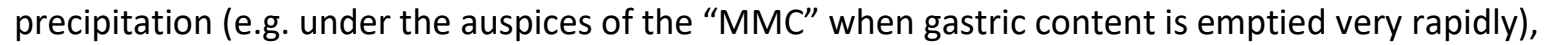
since precipitation rate has been reported to increase when media transfer is faster ${ }^{15}$. As there appears to be different sensitivities to gastric emptying time-frames among poorly soluble, weakly basic drugs, it is recommended to take a step-wise approach to characterize their behavior in vitro. First, conduct a two-stage test with buffers and biorelevant media, to determine whether bile salts and lecithin could have an impact on supersaturation and precipitation. Second, compare biorelevant two-stage and transfer experiments to determine whether gastric emptying rate plays a role in the supersaturation/precipitation behavior. If the time-frame of precipitation is affected by changes in the "gastric emptying rate", precipitation inhibitors could be screened for their ability to prolong supersaturation. 


\section{Conclusion}

By applying in vitro solubility, single and two-stage dissolution experiments of varying complexities, rDCS investigations correctly indicated the advantages of formulating ritonavir as an enabling ASD formulation, with a shift from class IIb (solubility limited absorption) to class Ila (dissolution limited absorption). Additionally, it was possible to identify a particle size target for the enabling formulation.

Furthermore, the use of different biorelevant media compositions over physiologically representative bile salt and lecithin ranges was shown to be a useful approach to assess luminal concentrations of ritonavir in the duodenum, as demonstrated for Norvir ${ }^{\circledR} 100 \mathrm{mg}$ tablet formulations. Coupling in vitro results with commercial PBPK modelling using the SIVA toolkit and the Simcyp Simulator, intraluminal duodenal concentrations as well as blood concentration-time profiles were predicted well.

Taken together, the rDCS and PBPK modelling approaches for ritonavir provide an illustrative example of the PBPK approach to understanding in vivo performance of enabling formulations. This approach should now be extended to a range of weakly basic compounds and enabling formulations to provide a global understanding of how poorly soluble weak bases behave after oral administration.

\section{Acknowledgements}

The Authors would like to thank Lee Ashworth and James Mann from AstraZeneca for providing the sampling cannulae for facilitated sampling for two-stage testing. 


\section{References}

1. Aulton ME, Taylor KMG. Aulton's Pharmaceutics E-Book: The Design and Manufacture of Medicines. 5th ed. London: Elsevier; 2017.

https://ebookcentral.proquest.com/lib/herts/detail.action?doclD=5253018.

2. DiMasi JA, Grabowski HG, Hansen RW. Innovation in the pharmaceutical industry: New estimates of R\&D costs. J Health Econ. 2016;47:20-33. doi:10.1016/j.jhealeco.2016.01.012

3. Fargen KM, Frei D, Fiorella D, et al. The FDA approval process for medical devices: an inherently flawed system or a valuable pathway for innovation? J Neurolnterventional Surg. 2013;5(4):269-275. doi:10.1136/neurintsurg-2012-010400

4. Kostewicz ES, Wunderlich M, Brauns U, Becker R, Bock T, Dressman JB. Predicting the precipitation of poorly soluble weak bases upon entry in the small intestine. J Pharm Pharmacol. 2004;56(1):43-51. doi:10.1211/0022357022511

5. Butler JM, Dressman JB. The Developability Classification System: Application of Biopharmaceutics Concepts to Formulation Development. J Pharm Sci. 2010;99(12):4940-4954. doi:10.1002/jps.22217

6. Rosenberger J, Butler J, Dressman J. A refined Developability Classification System (rDCS). J Pharm Sci. 2018;107. doi:10.1016/j.xphs.2018.03.030

7. Rosenberger J, Butler J, Muenster U, Dressman J. Application of a Refined Developability Classification System. J Pharm Sci. 2019;108(3):1090-1100. doi:10.1016/j.xphs.2018.10.044

8. Mann J, Dressman JB, Rosenblatt K, et al. Validation of Dissolution Testing with Biorelevant Media: an OrBiTo study. Mol Pharm. July 2017. doi:10.1021/acs.molpharmaceut.7b00198

9. Berben $\mathrm{P}, \mathrm{Ashworth} \mathrm{L}$, Beato $\mathrm{S}$, et al. Biorelevant dissolution testing of a weak base: Interlaboratory reproducibility and investigation of parameters controlling in vitro precipitation. Eur J Pharm Biopharm. 2019;140:141-148. doi:10.1016/j.ejpb.2019.04.017

10. Hansmann S, Miyaji Y, Dressman J. An in silico approach to determine challenges in the bioavailability of ciprofloxacin, a poorly soluble weak base with borderline solubility and permeability characteristics. Eur J Pharm Biopharm. 2018;122(Supplement C):186-196. doi:https://doi.org/10.1016/j.ejpb.2017.10.019

11. Hens B, Pathak SM, Mitra A, et al. In Silico Modeling Approach for the Evaluation of Gastrointestinal Dissolution, Supersaturation, and Precipitation of Posaconazole. Mol Pharm. 2017;14(12):4321-4333. doi:10.1021/acs.molpharmaceut.7b00396

12. Pathak SM, Ruff A, Kostewicz ES, Patel N, Turner DB, Jamei M. Model-Based Analysis of Biopharmaceutic Experiments To Improve Mechanistic Oral Absorption Modeling: An Integrated in Vitro in Vivo Extrapolation Perspective Using Ketoconazole as a Model Drug. Mol Pharm. 2017;14(12):4305-4320. doi:10.1021/acs.molpharmaceut.7b00406

13. Pathak SM, Schaefer KJ, Jamei M, Turner DB. Biopharmaceutic IVIVE-Mechanistic Modeling of Single- and Two-Phase In Vitro Experiments to Obtain Drug-Specific Parameters for Incorporation Into PBPK Models. J Pharm Sci. 2019;108(4):1604-1618.

doi:10.1016/j.xphs.2018.11.034 
14. United States Pharmacopeial Convention. United States Pharmacopeia and National Formulary (USP 41-NF 36) 41. Rockville, MD; 2016.

15. Ruff A, Fiolka T, Kostewicz ES. Prediction of Ketoconazole absorption using an updated in vitro transfer model coupled to physiologically based pharmacokinetic modelling. Eur J Pharm Sci. 2017;100:42-55. doi:http://dx.doi.org/10.1016/j.ejps.2016.12.017

16. Van Den Abeele et al. J. In vivo - in vitro investigation on the effect of reduced gastric acid secretion on the gastrointestinal disposition of a ritonavir ASD (Norvir ${ }^{\circledR}$ ) in fasted healthy volunteers. Submitt Abstr.

17. Ng J, Klein C, Chui Y, et al. The effect of food on ritonavir bioavailability following administration of ritonavir $100 \mathrm{mg}$ film-coated tablet in healthy adult subjects. J Int AIDS Soc. 2008;11(1):P247. doi:10.1186/1758-2652-11-S1-P247

18. Salem A, Chiu Y-L, Valdes J, Nilius A, Klein C. A novel ritonavir paediatric powder formulation is bioequivalent to ritonavir oral solution with a similar food effect. Antivir Ther. 2015;20.

doi:10.3851/IMP2932

19. Obach RS, Baxter JG, Liston TE, et al. The Prediction of Human Pharmacokinetic Parameters from Preclinical and <em>In Vitro</em > Metabolism Data. J Pharmacol Exp Ther. 1997;283(1):46.

20. Arora et al. S. Biopharmaceutic In Vitro In Vivo Extrapolation (IVIV_E) Informed PhysiologicallyBased Pharmacokinetic Model of Ritonavir Norvir ${ }^{\circledast}$ Tablet Absorption in Humans Under Fasted and Fed State Conditions. Mol Pharm (Submitted).

21. Law D, Krill SL, Schmitt EA, et al. Physicochemical considerations in the preparation of amorphous ritonavir-poly(ethylene glycol) 8000 solid dispersions. J Pharm Sci. 2001;90(8):1015-1025. doi:10.1002/jps.1054

22. Law D, Schmitt EA, Marsh KC, et al. Ritonavir-PEG 8000Amorphous Solid Dispersions: In vitro and In vivo Evaluations. J Pharm Sci. 2004;93(3):563-570. doi:10.1002/jps.10566

23. Xu H, Vela S, Shi Y, Marroum P, Gao P. In Vitro Characterization of Ritonavir Drug Products and Correlation to Human in Vivo Performance. Mol Pharm. 2017;14(11):3801-3814. doi:10.1021/acs.molpharmaceut.7b00552

24. Ellenberger DJ, Miller DA, Kucera SU, Williams RO. Generation of a Weakly Acidic Amorphous Solid Dispersion of the Weak Base Ritonavir with Equivalent In Vitro and In Vivo Performance to Norvir Tablet. AAPS PharmSciTech. 2018;19(5):1985-1997. doi:10.1208/s12249-018-1060-x

25. Indulkar AS, Gao Y, Raina SA, Zhang GGZ, Taylor LS. Crystallization from Supersaturated Solutions: Role of Lecithin and Composite Simulated Intestinal Fluid. Pharm Res. 2018;35(8):158. doi:10.1007/s11095-018-2441-2

26. Indulkar AS, Lou X, Zhang GGZ, Taylor LS. Insights into the Dissolution Mechanism of RitonavirCopovidone Amorphous Solid Dispersions: Importance of Congruent Release for Enhanced Performance. Mol Pharm. 2019;16(3):1327-1339. doi:10.1021/acs.molpharmaceut.8b01261

27. Hsu A, Granneman GR, Witt G, et al. Multiple-dose pharmacokinetics of ritonavir in human immunodeficiency virus-infected subjects. Antimicrob Agents Chemother. 1997;41(5):898. doi:10.1128/AAC.41.5.898 
28. Umehara K, Huth F, Won CS, Heimbach T, He H. Verification of a physiologically based pharmacokinetic model of ritonavir to estimate drug-drug interaction potential of CYP3A4 substrates. Biopharm Drug Dispos. 2018;39(3):152-163. doi:10.1002/bdd.2122

29. Koudriakova T, latsimirskaia E, Utkin I, et al. Metabolism of the Human Immunodeficiency Virus Protease Inhibitors Indinavir and Ritonavir by Human Intestinal Microsomes and Expressed Cytochrome P4503A4/3A5: Mechanism-Based Inactivation of Cytochrome P4503A by Ritonavir. Drug Metab Dispos. 1998;26(6):552.

30. Denissen JF, Grabowski BA, Johnson MK, et al. Metabolism And Disposition of the HIV-1 Protease Inhibitor Ritonavir (ABT-538) in Rats, Dogs, and Humans. Drug Metab Dispos. 1997;25(4):489.

31. Mithani SD, Bakatselou V, TenHoor CN, Dressman JB. Estimation of the Increase in Solubility of Drugs as a Function of Bile Salt Concentration. Pharm Res. 1996;13(1):163-167. doi:10.1023/A:1016062224568

32. Galia E, Nikolaidi E, Hörter D, Löbenberg R, Reppas C, Dressman JB. Evaluation of Various Dissolution Media for Predicting In Vivo Performance of Class I and II Drugs. Pharm Res. 1998;15:698-705. doi:10.1023/A:1011910801212

33. Markopoulos C, Andreas CJ, Vertzoni M, Dressman J, Reppas C. In-vitro simulation of luminal conditions for evaluation of performance of oral drug products: Choosing the appropriate test media. Eur J Pharm Biopharm. 2015;93:173-182. doi:10.1016/j.ejpb.2015.03.009

34. Dressman JB, Berardi RR, Dermentzoglou LC, et al. Upper gastrointestinal (GI) pH in young, healthy men and women. Pharm Res. 1990;7(7):756-761.

35. Dressman JB, Vertzoni M, Goumas K, Reppas C. Estimating drug solubility in the gastrointestinal tract. Drug Solubility Meas It Improve It. 2007;59(7):591-602. doi:10.1016/j.addr.2007.05.009

36. Ghadi R, Dand N. BCS class IV drugs: Highly notorious candidates for formulation development. J Controlled Release. 2017;248:71-95. doi:10.1016/j.jconrel.2017.01.014

37. Savla R, Browne J, Plassat V, Wasan KM, Wasan EK. Review and analysis of FDA approved drugs using lipid-based formulations. Drug Dev Ind Pharm. 2017;43(11):1743-1758. doi:10.1080/03639045.2017.1342654

38. European Medicines Agency - Summary of product characteristics Norvir 100 mg tablet. https://www.ema.europa.eu > norvir-epar-product-information_en.

39. Wuyts B, Brouwers J, Mols R, Tack J, Annaert P, Augustijns P. Solubility Profiling of HIV Protease Inhibitors in Human Intestinal Fluids. J Pharm Sci. 2013;102(10):3800-3807. doi:10.1002/jps.23698

40. Clarysse S, Brouwers J, Tack J, Annaert P, Augustijns P. Intestinal drug solubility estimation based on simulated intestinal fluids: Comparison with solubility in human intestinal fluids. Eur $J$ Pharm Sci. 2011;43(4):260-269. doi:10.1016/j.ejps.2011.04.016

41. He Y, Ho C. Amorphous Solid Dispersions: Utilization and Challenges in Drug Discovery and Development. J Pharm Sci. 2015;104(10):3237-3258. doi:10.1002/jps.24541 
42. Fuchs A, Dressman JB. Composition and Physicochemical Properties of Fasted-State Human Duodenal and Jejunal Fluid: A Critical Evaluation of the Available Data. J Pharm Sci. 2014;103(11):3398-3411. doi:10.1002/jps.24183

43. Litou C. Investigation of the influence of pre- and post-absorptive factors on the pharmacokinetic behaviour of anti-HIV medicines using PBPK modeling: Case example - bioenabling formulation of etravirine. Eur J Pharm Sci.

44. Berlin M, Przyklenk K-H, Richtberg A, Baumann W, Dressman JB. Prediction of oral absorption of cinnarizine - A highly supersaturating poorly soluble weak base with borderline permeability. Eur J Pharm Biopharm. 2014;88(3):795-806. doi:http://dx.doi.org/10.1016/j.ejpb.2014.08.011

45. Berlin M, Ruff A, Kesisoglou F, Xu W, Wang MH, Dressman JB. Advances and challenges in PBPK modeling - Analysis of factors contributing to the oral absorption of atazanavir, a poorly soluble weak base. Eur J Pharm Biopharm. 2015;93:267-280. doi:10.1016/j.ejpb.2015.03.031 